Limits on the flux of tau neutrinos from $1 \mathrm{PeV}$ to $3 \mathrm{EeV}$ with the MAGIC telescopes

M.L. Ahnen, S. Ansoldi , L.A. Antonelli, C. Arcaro, D. Baack, A. Babić, B. Banerjee, P. Bangale, U. Barres de Almeida, J.A. Barrio, J. Becerra González, W. Bednarek, E. Bernardini, R. Ch. Berse,

A. Berti, W. Bhattacharyya, A. Biland, O. Blanch, G. Bonnoli, R. Carosi, A. Carosi, G. Ceribella, A. Chatterjee, S.M. Colak, P. Colin, E. Colombo, J.L. Contreras, J. Cortina, S. Covino,

P. Cumani, P. Da Vela, F. Dazzi, A. De Angelis, B. De Lotto, M. Delfino, J. Delgado, F. Di Pierro, A. Domínguez,

D. Dominis Prester, D. Dorner, M. Doro, S. Einecke, D. Elsaesser, V. Fallah Ramazani, A. Fernández-Barral, D. Fidalgo, M.V. Fonseca,

L. Font, C. Fruck, D. Galindo, R. J. García López, M. Garczarczyk, M. Gaug, P. Giammaria, N. Godinović, D. Góra, D. Guberman, D. Hadasch , A. Hahn, T. Hassan, M. Hayashida, J. Herrera,

J. Hose, D. Hrupec, K. Ishio, Y. Konno , H. Kubo , J. Kushida,

D. Kuveždić, D. Lelas, E. Lindfors, S. Lombardi, F. Longo, M. López,

C. Maggio, P. Majumdar, M. Makariev, G. Maneva, M. Manganaro,

K. Mannheim, L. Maraschi, M. Mariotti, M. Martínez, S. Masuda,

D. Mazin , K. Mielke, M. Minev, J.M. Miranda, R. Mirzoyan,

A. Moralejo, V. Moreno, E. Moretti, T. Nagayoshi , V. Neustroev, A. Niedzwiecki, M. Nievas Rosillo, C. Nigro, K. Nilsson, D. Ninci, K. Nishijima , K. Noda, L. Nogués, S. Paiano, J. Palacio, D. Paneque, R. Paoletti, J.M. Paredes, G. Pedaletti, M. Peresano, M. Persic , P. G. Prada Moroni, E. Prandini, I. Puljak, J.R. Garcia, I. Reichardt, W. Rhode, M. Ribó, J. Rico, C. Righi, A. Rugliancich, T. Saito, K. Satalecka, T. Schweizer, J. Sitarek , I. Šnidarić, D. Sobczynska, A. Stamerra, M. Strzys, T. Surić, M. Takahashi, L. Takalo, F. Tavecchio, P. Temnikov, T. Terzić, M. Teshima, N. Torres-Albà, A. Treves, S. Tsujimoto, G. Vanzo, M. Vazquez Acosta, I. Vovk, J.E. Ward, M. Will, D. Zarić

PII:

DOI:

Reference:

To appear in:

Received date: Revised date: Accepted date:
S0927-6505(18)30013-6

10.1016/j.astropartphys.2018.05.002

ASTPHY 2294

\section{Astroparticle Physics}

9 January 2018

2 May 2018

7 May 2018

Please cite this article as: M.L. Ahnen, S. Ansoldi, L.A. Antonelli, C. Arcaro, D. Baack, A. Babić, B. Banerjee, P. Bangale, U. Barres de Almeida, J.A. Barrio, J. Becerra González, W. Bednarek, E. Bernardini, R. Ch. Berse, A. Berti, W. Bhattacharyya, A. Biland, O. Blanch, G. Bonnoli, R. Carosi, A. Carosi, G. Ceribella, A. Chatterjee, S.M. Colak, P. Colin, E. Colombo, J.L. Contreras, J. Cortina, S. Covino, P. Cumani, P. Da Vela, F. Dazzi, A. De Angelis, B. De Lotto, M. Delfino, J. Delgado, 
F. Di Pierro, A. Domínguez, D. Dominis Prester, D. Dorner, M. Doro, S. Einecke, D. Elsaesser, V. Fallah Ramazani, A. Fernández-Barral, D. Fidalgo, M.V. Fonseca, L. Font, C. Fruck, D. Galindo, R. J. García López, M. Garczarczyk, M. Gaug, P. Giammaria, N. Godinović, D. Góra, D. Guberman, D. Hadasch , A. Hahn, T. Hassan, M. Hayashida, J. Herrera, J. Hose, D. Hrupec, K. Ishio, Y. Konno, H. Kubo, J. Kushida, D. Kuveždić, D. Lelas, E. Lindfors, S. Lombardi, F. Longo, M. López, C. Maggio, P. Majumdar, M. Makariev, G. Maneva, M. Manganaro, K. Mannheim, L. Maraschi, M. Mariotti, M. Martínez, S. Masuda, D. Mazin , K. Mielke, M. Minev, J.M. Miranda, R. Mirzoyan, A. Moralejo, V. Moreno, E. Moretti, T. Nagayoshi, V. Neustroev, A. Niedzwiecki, M. Nievas Rosillo, C. Nigro, K. Nilsson, D. Ninci, K. Nishijima, K. Noda, L. Nogués, S. Paiano, J. Palacio, D. Paneque, R. Paoletti, J.M. Paredes, G. Pedaletti, M. Peresano, M. Persic , P. G. Prada Moroni, E. Prandini, I. Puljak, J.R. Garcia, I. Reichardt, W. Rhode, M. Ribó, J. Rico, C. Righi, A. Rugliancich, T. Saito, K. Satalecka, T. Schweizer, J. Sitarek, I. Šnidarić, D. Sobczynska, A. Stamerra, M. Strzys, T. Surić, M. Takahashi, L. Takalo, F. Tavecchio, P. Temnikov, T. Terzić, M. Teshima, N. Torres-Albà, A. Treves, S. Tsujimoto, G. Vanzo, M. Vazquez Acosta, I. Vovk, J.E. Ward, M. Will, D. Zarić, Limits on the flux of tau neutrinos from $1 \mathrm{PeV}$ to $3 \mathrm{EeV}$ with the MAGIC telescopes, Astroparticle Physics (2018), doi: 10.1016/j.astropartphys.2018.05.002

This is a PDF file of an unedited manuscript that has been accepted for publication. As a service to our customers we are providing this early version of the manuscript. The manuscript will undergo copyediting, typesetting, and review of the resulting proof before it is published in its final form. Please note that during the production process errors may be discovered which could affect the content, and all legal disclaimers that apply to the journal pertain. 


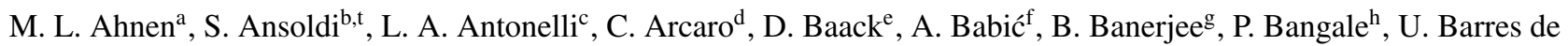
Almeida $^{\mathrm{h}, \mathrm{i}}$, J. A. Barrio ${ }^{\mathrm{j}}$, J. Becerra González ${ }^{\mathrm{k}}$, W. Bednarek ${ }^{\mathrm{l}}$, E. Bernardini ${ }^{\mathrm{d}, \mathrm{m}, \mathrm{w}}$, R. Ch. Berse ${ }^{\mathrm{e}}$, A. Berti ${ }^{\mathrm{b}, \mathrm{x}}$, W. Bhattacharyya ${ }^{\mathrm{m}}$, A. Biland ${ }^{\mathrm{a}}$, O. Blanch ${ }^{\mathrm{n}}$, G. Bonnoli ${ }^{\mathrm{o}}$, R. Carosi ${ }^{\mathrm{o}}$, A. Carosic ${ }^{\mathrm{c}}$, G. Ceribella ${ }^{\mathrm{h}}$, A. Chatterjee ${ }^{\mathrm{g}}$, S. M. Colak ${ }^{\mathrm{n}}$, P. Colin $^{\mathrm{h}}$, E. Colombo $^{\mathrm{k}}$, J. L. Contreras ${ }^{j}$, J. Cortina $^{\mathrm{n}}$, S. Covino ${ }^{\mathrm{c}}$, P. Cumani ${ }^{\mathrm{n}}$, P. Da Vela ${ }^{\mathrm{o}}$, F. Dazzi ${ }^{\mathrm{c}}$, A. De Angelis ${ }^{\mathrm{d}}$, B. De Lotto ${ }^{\mathrm{b}}$, M. Delfino $^{\mathrm{n}, \mathrm{y}}$,

J. Delgado ${ }^{n}$, F. Di Pierro ${ }^{d}$, A. Domínguez ${ }^{j}$, D. Dominis Prester ${ }^{\mathrm{f}}$, D. Dorner ${ }^{\mathrm{p}}$, M. Doro ${ }^{\mathrm{d}}$, S. Einecke , D. Elsaesser $^{\mathrm{e}}$, V. Fallah Ramazani $^{\mathrm{q}}$, A. Fernández-Barral ${ }^{\mathrm{n}}$, D. Fidalgo ${ }^{\mathrm{j}}$, M. V. Fonseca ${ }^{\mathrm{j}}$, L. Font $^{\mathrm{r}}$, C. Fruck ${ }^{\mathrm{h}}$, D. Galindo ${ }^{\mathrm{s}}$, R. J. García López ${ }^{\mathrm{k}}$,

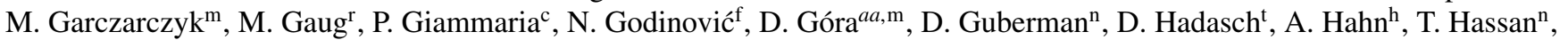

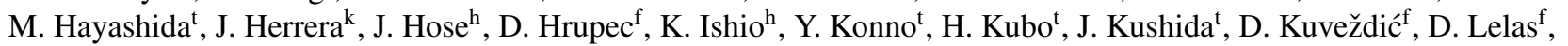
E. Lindfors ${ }^{\mathrm{q}}$, S. Lombardic ${ }^{\mathrm{c}}$, F. Longo ${ }^{\mathrm{c}, \mathrm{x}}$, M. López ${ }^{\mathrm{j}}$, C. Maggio ${ }^{\mathrm{r}}$, P. Majumdar ${ }^{\mathrm{g}}$, M. Makariev ${ }^{\mathrm{u}}$, G. Maneva $^{\mathrm{u}}$, M. Manganaro $^{\mathrm{k}}$, K. Mannheim ${ }^{\mathrm{p}}$, L. Maraschic ${ }^{\mathrm{c}}$, M. Mariotti ${ }^{\mathrm{d}}$, M. Martínez ${ }^{\mathrm{n}}$, S. Masuda ${ }^{\mathrm{t}}$, D. Mazin ${ }^{\mathrm{h}, \mathrm{t}}$, K. Mielke ${ }^{\mathrm{e}}$, M. Minev $^{\mathrm{u}}, \mathrm{J}$. M. Miranda ${ }^{\mathrm{o}}$,

R. Mirzoyan ${ }^{\mathrm{h}}$, A. Moralejo $^{\mathrm{n}}$, V. Moreno ${ }^{\mathrm{r}}$, E. Moretti ${ }^{\mathrm{h}}$, T. Nagayoshi ${ }^{\mathrm{t}}$, V. Neustroev ${ }^{\mathrm{q}}$, A. Niedzwiecki ${ }^{1}$, M. Nievas Rosillo ${ }^{\mathrm{j}}$,

C. Nigrom ${ }^{\mathrm{m}}$, K. Nilsson ${ }^{\mathrm{q}}$, D. Ninci ${ }^{\mathrm{n}}$, K. Nishijima ${ }^{\mathrm{t}}$, K. Noda ${ }^{\mathrm{n}}$, L. Nogués ${ }^{\mathrm{n}}$, S. Paiano ${ }^{\mathrm{d}}$, J. Palacio ${ }^{\mathrm{n}}$, D. Paneque ${ }^{\mathrm{h}}$, R. Paoletti ${ }^{\mathrm{e}}$,

J. M. Paredes ${ }^{\text {s }}$, G. Pedaletti ${ }^{\mathrm{m}}$, M. Peresano ${ }^{\mathrm{b}}$, M. Persic ${ }^{\mathrm{b}, \mathrm{z}}$, P. G. Prada Moroni ${ }^{\mathrm{v}}$, E. Prandini ${ }^{\mathrm{d}}$, I. Puljak ${ }^{\mathrm{f}}$, J. R. Garcia ${ }^{\mathrm{h}}$, I. Reichardt ${ }^{\mathrm{d}}$,

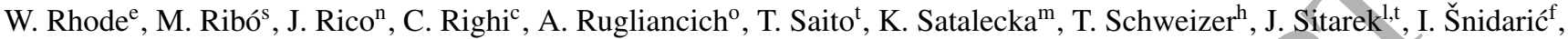

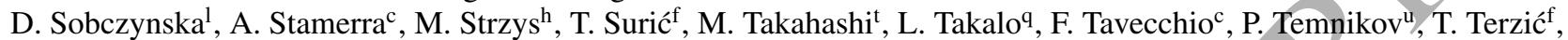

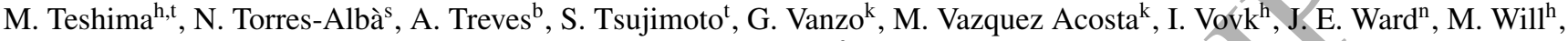
D. Zarić ${ }^{\mathrm{f}}$

${ }^{a}$ ETH Zurich, CH-8093 Zurich, Switzerland

${ }^{b}$ Università di Udine, and INFN Trieste, I-33100 Udine, Italy

${ }^{c}$ National Institute for Astrophysics (INAF), I-00136 Rome, Italy

${ }^{d}$ Università di Padova and INFN, I-35131 Padova, Italy

${ }^{e}$ Technische Universität Dortmund, D-44221 Dortmund, Germany

${ }^{f}$ Croatian MAGIC Consortium: University of Rijeka, 51000 Rijeka, University of Split - FESB, 21000 Split, University of Zagreb - FER, 10000 Zagreb, University of Osijek, 31000 Osijek and Rudjer Boskovic Institute, 10000 Zagreb, Croatia.

${ }^{g}$ Saha Institute of Nuclear Physics, HBNI, 1/AF Bidhannagar, Salt Lake, Sector-1, Kolkata 700064, India

${ }^{h}$ Max-Planck-Institut für Physik, D-80805 München, Germany

${ }^{i}$ now at Centro Brasileiro de Pesquisas Físicas (CBPF), 22290-180 URCA, Rio de Janeiro (RJ), Brasil

${ }^{j}$ Unidad de Partículas y Cosmología (UPARCOS), Universidad Complutense, E-28040 Madrid, Spain

${ }^{k}$ Inst. de Astrofísica de Canarias, E-38200 La Laguna, and Universidad de La Laguna, Dpto. Astrofísica, E-38206 La Laguna, Tenerife, Spain

${ }^{l}$ University of Łódź, Department of Astrophysics, PL-90236 Łódź, Poland

${ }^{m}$ Deutsches Elektronen-Synchrotron (DESY), D-15738 Zeuthen, Germany

${ }^{n}$ Institut de Física d'Altes Energies (IFAE), The Barcelona Institute of Science and Technology (BIST), E-08193 Bellaterra (Barcelona), Spain

${ }^{\circ}$ Università di Siena, and INFN Pisa, I-53100 Siena, Italy

${ }^{p}$ Universität Würzburg, D-97074 Würzburg, Germany

${ }^{q}$ Finnish MAGIC Consortium: Tuorla Observatory and Finnish Centre of Astronomy with ESO (FINCA), University of Turku, Vaisalantie 20, FI-21500 Piikkiö, Astronomy Division, University of Oulu, FIN-90014 University of Oulu, Finland

${ }^{r}$ Departament de Física, and CERES-IEEC, Universitat Autónoma de Barcelona, E-08193 Bellaterra, Spain

${ }^{s}$ Universitat de Barcelona, ICC, IEEC-UB, E-08028 Barcelona, Spain

${ }^{t}$ Japanese MAGIC Consortium: ICRR, The University of Tokyo, 277-8582 Chiba, Japan; Department of Physics, Kyoto University, 606-8502 Kyoto, Japan; Tokai University, 259-1292 Kanagawa, Japan; The University of Tokushima, 770-8502 Tokushima, Japan

unst. for Nucl. Research and Nucl. Energy, Bulgarian Academy of Sciences, BG-1784 Sofia, Bulgaria

$\checkmark$ Università di Pisa, and INFN Pisa, I-56126 Pisa, Italy

${ }^{w}$ Humboldt University of Berlin, Institut für Physik D-12489 Berlin Germany

${ }^{x}$ also at Dipartimento di Fisica, Università di Trieste, I-34127 Trieste, Italy

${ }^{y}$ also at Port d'Informació Científica (PIC) E-08193 Bellaterra (Barcelona) Spain

zalso at INAF-Trieste and Dept. of Physics $\mathcal{E}$ Astronomy, University of Bologna

also at Institute of Nuclear Physics PAS, Radzikowskiego 152, Cracow, Poland

\begin{abstract}
A search for tau neutrino induced showers with the MAGIC telescopes is presented. The MAGIC telescopes located at an altitude of $2200 \mathrm{~m}$ a.s.l. in the Canary Island of La Palma, can point towards the horizon or a few degrees below across an azimuthal range of about 80 degrees. This provides a possibility to search for air showers induced by tau leptons arising from interactions of tau neutrinos in the Earth crust or the surrounding ocean. In this paper we show how such air showers can be discriminated from the background of very inclined hadronic showers by using Monte Carlo simulations. Taking into account the orography of the site, the point source acceptance and the event rates expected have been calculated for a sample of generic neutrino fluxes from photo-hadronic interactions in AGNs. The analysis of about 30 hours of data taken towards the sea leads to a $90 \%$ C.L. point source limit for tau neutrinos in the energy range from $1.0 \times 10^{15} \mathrm{eV}$ to $3.0 \times 10^{18} \mathrm{eV}$ of about $E_{v_{\tau}}^{2} \times \phi\left(E_{v_{\tau}}\right)<2.0 \times 10^{-4} \mathrm{GeV} \mathrm{cm}^{-2} \mathrm{~s}^{-1}$ for an assumed power-law neutrino spectrum with spectral index $\gamma=-2$. However, with 300 hours and in case of an optimistic neutrino flare model, limits of the level down to $E_{v_{\tau}}^{2} \times \phi\left(E_{\gamma_{\tau}}\right)<8.4 \times 10^{-6} \mathrm{GeV} \mathrm{cm}^{-2} \mathrm{~s}^{-1}$ can be expected.
\end{abstract}

Keywords: Gamma-ray astronomy, Cherenkov telescopes, tau neutrinos 


\section{Introduction}

The discovery of an astrophysical flux of high-energy neutrinos by IceCube [1] was a major step forward in the ongoing search for the origin of cosmic rays, since neutrino emission needs to be produced by hadronic interactions in astrophysical accelerators. The observed neutrino flux by IceCube and its compostion is in agreement with equal fractions of all neutrino flavours $[2,3]$. Tau neutrinos in the IceCube flux should also be expected, due to neutrino oscillation, but up to now, $v_{\tau}$ have not been identified.

The detection of $v_{\tau}$ is very important from both the astrophysical and particle physics point of view. It would give new information about the astrophysical $v_{\tau}$ flux and serve as an additional confirmation of the astrophysical origin of the IceCube high energy diffuse neutrino signal $[2,3]$. It also would shed light on the emission mechanisms at the source, test the fundamental properties of neutrinos over extremely long baselines, and better constrain new physics models which predict significant deviations from equal fractions of all flavors.

Large detectors like IceCube ${ }^{1}$, ANTARES ${ }^{2}$, the Pierre Auger Observatory [4], the Telescope Array ${ }^{3}$, or radio detectors like ANITA [5], have the capability to detect neutrino induced showers. Especially if tau neutrinos interact close to the Earth surface, the so-called Earth's skimming neutrinos $[6,7,8]$ can produce tau leptons which can emerge from the Earth, decay and produce extended air showers. If the decay vertex of a tau lepton is close enough to a surface detector, it can be detected and distinguished from very inclined proton and nuclei induced showers due to the presence of its electromagnetic component.

Above PeV energies, the Earth becomes opaque to electron and muon neutrinos, while the tau neutrino flux is regenerated through subsequent tau lepton decays to neutrinos. At highenergies, the tau neutrino interacts in the Earth producing a tau lepton which in turn decays into a $v_{\tau}$ with lower energy due to its short lifetime. The regeneration chain $v_{\tau} \rightarrow \tau \rightarrow v_{\tau} \ldots$ continues until the tau lepton reaches the detector. This effect can lead to a significant enhancement of the tau lepton flux of up to about $40 \%$ more than the initial cosmic flux of tau neutrinos of energies between 1-100 PeV $[9,10]$.

Tau neutrinos that pass through the earth crust, are the only ones that can produce particle showers that can be detected by Imaging Atmospheric Cherenkov Telescopes (IACTs). In the interaction all neutrino types loose part of their energy due to charged current and neutral current interactions. In the case of $v_{\mathrm{e}}$ 's crossing the Earth, an electron is produced which is rapidly brought to rest in matter. In the case of muon neutrinos $v_{\mu}$ 's, a muon is created which subsequently decays. However, before decaying the muon propagates through matter, too. As its radiation length in matter is much smaller than its decay length at our energies of interest, it loses most of its energy. The pro-

\footnotetext{
${ }^{*}$ Corresponding authors: Dariusz Gora (dariusz.gora@ifj.edu.pl) and Elisa Bernardini (Elisa.Bernardini@desy.de)

${ }^{1}$ http://icecube.wisc.edu

${ }^{2}$ http://antares.in2p3.fr

${ }^{3}$ http://www.telescopearray.org
}

duced muon can occasionally escape from the Earth, but it decays only rarely in the atmosphere since the decay length is about $10^{8}$ times larger than the one of a tau lepton ${ }^{4}$. Energetic tau neutrinos, produce extended air showers at the decay of the tau-particle, which itself loses much less energy while it travels through matter and which can be efficiently detected by IACTs and discriminated against background. Thus the earthskimming method is suitable for the detection of tau neutrinos ${ }^{5}$.

To detect neutrino-induced showers with an IACT system, it needs to be pointed towards the ground, e.g. the side of a mountain or the sea surface $[6,11,12,13,14]$.

In this paper we present limits on the flux of tau neutrinos from the MAGIC telescopes. MAGIC is located at the Roque de los Muchachos Observatory at an altitude of about $2200 \mathrm{~m}$ above sea level $\left(28.8^{\circ} \mathrm{N}, 17.9^{\circ} \mathrm{W}\right)$, in the Canary Island of La Palma (Spain). The observatory consists of two telescopes placed at a distance of $85 \mathrm{~m}$ from one another. The MAGIC telescopes have a mirror of $17 \mathrm{~m}$ diameter and a field of view (FOV) of $3.5^{\circ}$. They have been built to detect cosmic $\gamma$-rays in the energy range from $\sim 50 \mathrm{GeV}$ to $\sim 50 \mathrm{TeV}$ [15]. For this search, MAGIC was used as a neutrino detector, in order to look for air showers induced by tau neutrinos ( $\tau$-induced showers) in the PeV to EeV energy range. Here we report final results of our preliminary studies presented in $[16,17]$.

The search of tau neutrinos with MAGIC is performed pointing the telescopes in the direction of $v_{\tau}$ which escape the Earth crust and later cross the ocean (see Figure 1, left). The telescopes can point up to 6 degrees below the horizontal plane, covering an azimuthal range of 80 degrees (see Figure 1, right). The location of MAGIC contains the right distance of the telescope to the average point of the tau lepton decay vertex. This distance should be at least a few tens of times larger than the decay length of the tau lepton. At $10 / 1000 \mathrm{PeV}$ the tau decay length is about $0.5 / 50 \mathrm{~km}$. If such a condition is not fulfilled, the induced air-shower is not fully developed, leading to a too small amount of produced Cherenkov light reaching the Cherenkov telescopes.

In [18], the effective area for up-going tau neutrino observations for MAGIC was calculated analytically and found to reach $5 \cdot 10^{5} \mathrm{~m}^{2}$ at $100 \mathrm{EeV}$. An analytical approximation results in tau neutrino effective areas from $\sim 10^{3} \mathrm{~m}^{2}$ (at $100 \mathrm{TeV}$ ) to $6 \times 10^{4} \mathrm{~m}^{2}$ (at $300 \mathrm{PeV}$ ) for an observation angle of about $1.5^{\circ}$ below the horizon ${ }^{6}$, rapidly diminishing with larger inclination. However, the sensitivity for diffuse neutrinos was found to be very poor compared to the IceCube or Pierre Auger experiments due to the limited FOV, and the shorter observation times with MAGIC.

In the case the telescopes are pointed to flaring or disrupting point sources such as gamma ray bursts (GRBs) or active galactic nuclei (AGNs), one can expect to observe a signal from

\footnotetext{
${ }^{4}$ The muon decay length is about $6.6 \times 10^{6} \mathrm{~km}$ for a muon energy of $1 \mathrm{PeV}$.

${ }^{5}$ The flux of $\mu$ leptons produced due to charged current interaction of $v_{\mu}$ is more than one order of magnitude lower than for $\tau$ leptons [79, 80].

${ }^{6} \mathrm{In}[18]$ the fluorescence emission above $300 \mathrm{PeV}$ was also included in the aperture calculation, thus the aperture can reach about $2 \times 10^{5} \mathrm{~m}^{2}$ at $1 \mathrm{EeV}$. In this work we do not included fluorescence emission.
} 

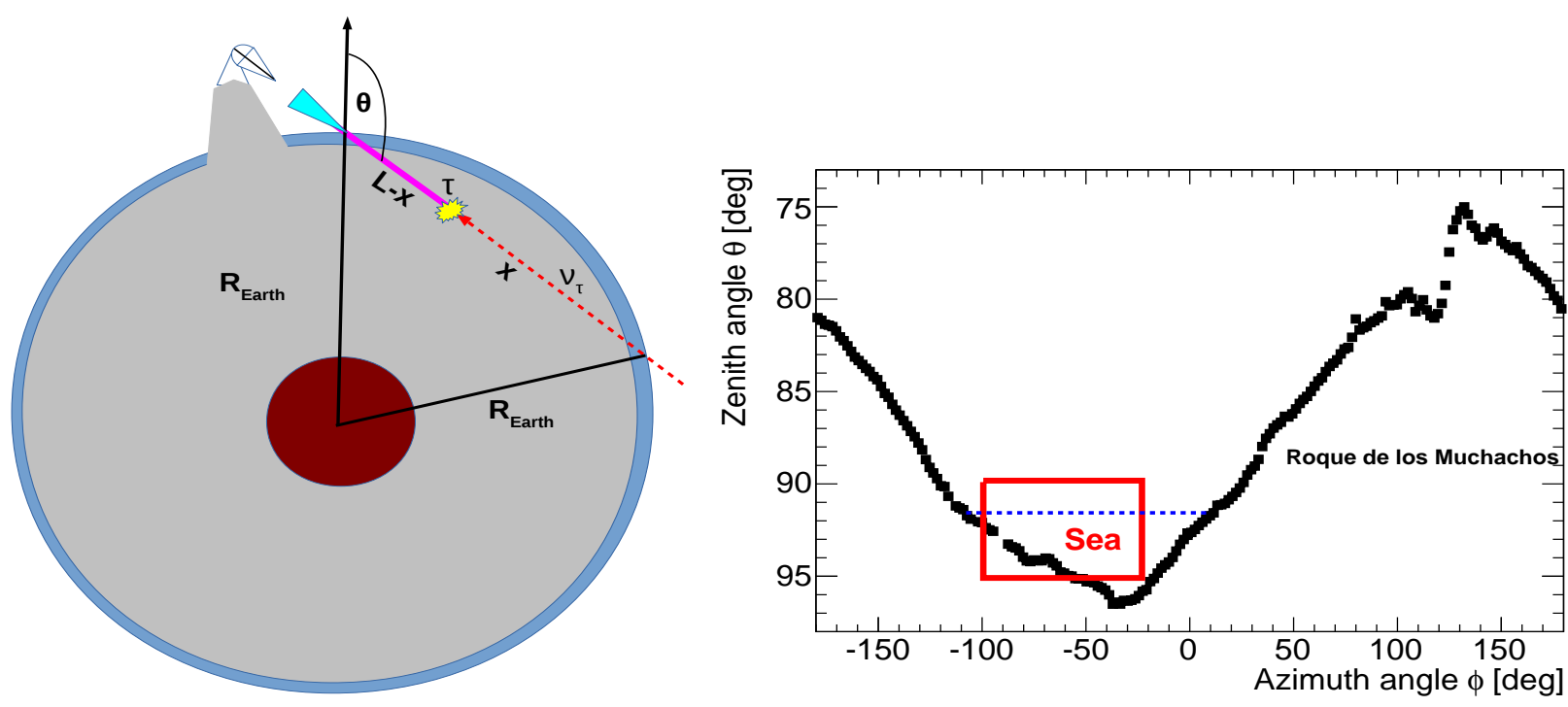

Figure 1: Left panel: Illustration of a neutrino converting after distance $x$ into a tau lepton inside the Earth with radius $R_{\text {Earth }}$ and crossing the layer of the Sea towards the MAGIC telescopes. Right panel: the horizon seen from the MAGIC telescopes. The region with azimuth $\phi$ in the range from -20 deg to -100 deg and zenith angle $\theta$ from $\sim 90 \mathrm{deg}$ to $\sim 95 \mathrm{deg}$ can be used to point toward the sea. The region with azimuth from $-20 \mathrm{deg}$ to 0 deg is excluded, due to shadowing by the telescopes access towers.

Table 1: Summary of the data taken at very large-zenith angles during MAGICobservations from October 2015 to March 2017.

\begin{tabular}{|c|c|c|c|}
\hline & seaOFF seaON & Roque & HET \\
\hline Zenith angle $\theta\left(^{\circ}\right)$ & 92.5 & 89.5 & $85-93$ \\
\hline Azimuth $\phi\left(^{\circ}\right)$ & -30 & 170 & $-80-(-75)$ \\
\hline Observation time (h) & 31.5 & 7.5 & 4.5 \\
\hline
\end{tabular}

neutrinos. Indeed, it was shown by the Ashra (All-sky Survey High Resolution Air-shower detector) team [11] and by [18], that Cherenkov telescopes can be sensitive to close-by GRBs $(z<0.1)$. It is also known that a large amount of rock surrounding the site, like mountains, can lead to a significant enhancement of the tau lepton flux, see for example [13]. However, in the case of the MAGIC site, the mountain is too close to the telescopes, and the possible $\tau$-leptons emerging from the mountain would not have sufficient time to create the electromagnetic showers before reaching the telescopes.

It is worth to mention that this kind of observations can be performed during the presence of high clouds above the detector. In such a case, the regular MAGIC gamma-ray observations are not possible, but such conditions allow to perform horizontal observations for tau neutrinos. The amount of observation time varies from one to another MAGIC observation season, but amounts to about 100 hours per year [19].

The structure of this paper is the following: Section 2 describes the recent MAGIC observation at very large zenith angles $\left(>85^{\circ}\right)$ and presents the Monte Carlo (MC) simulation chain. In Section 3, we study the properties of shower images on the camera focal plane from $\tau$-induced showers, and we show that MAGIC can discriminate $\tau$-induced showers from the background of large-zenith angle cosmic-ray (CR) induced showers. In Section 4 and 5 details of the acceptance calculations are presented, together with the expected event rates and the MAGIC sensitivity for tau neutrinos. Finally, in Section 6, a short summary is given.

\section{MAGIC observations and Monte Carlo simulations}

The MAGIC telescopes have collected approximately 30 hours of data at very large zenith angles $\left(\theta=92.5^{\circ}\right)$ in the direction of Sea, referred to as seaON. Events from slightly above the sea (seaOFF), towards the Roque de los Muchachos mountain or pointing to the Highest Energy Track (HET) [20] event from IceCube $^{7}$ have also been obtained. Details about all event samples are shown in Table 1. A significant amount of data, about $91 \%$, was accumulated during the presence of high clouds at the MAGIC site.

${ }^{7}$ Up to now, the HET is the highest energy neutrino event seen in the IceCube data, with a pointing accuracy of about $0.27^{\circ}$ (median), thus particularly interesting for IACTs, given their FOV of a few degrees. 


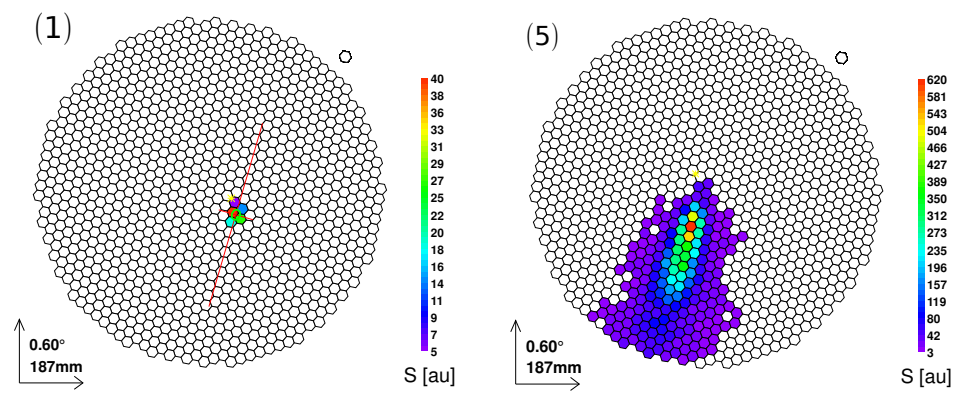

Figure 2: Examples of simulated events triggered stereoscopically and shower images for $1 \mathrm{PeV}$ protons/muon injected at the top of the atmosphere under a zenith angle of $\theta \simeq 86^{\circ}$ (background expectation), as seen by one of the MAGIC telescopes. The first interaction point is at a vertical depth, defined as the starting altitude in $\mathrm{g} / \mathrm{cm}^{2}$ of the mass overburden of the atmosphere, below $50 \mathrm{~g} / \mathrm{cm}^{2}$ and a dístance between the detector and the proton interaction point of about $800 \mathrm{~km}$. Example of (1) CR showers at lower energies (see text), (2) a single 1 PeV muon as the primary particle, injected at the top of the atmosphere, interacting via radiative processes and producing a bright gamma-like image on the camera.

The rate of events seen by both MAGIC telescopes the socalled stereo event rate, in case of seaOFF data, due to increasing attenuation of the Cherenkov light as a function of the zenith angle, is about 27 times larger $(\sim 4.6 \mathrm{~Hz})$ than for seaON $(\sim 0.17 \mathrm{~Hz})$ observations. Thus, seaOFF observations provide a high-statistics background estimate for the seaON data. In principle, the expected seaON background should be almost zero. However, due to the specific location of MAGIC, the curvature of Earth and the fact that the MAGIC telescopes can point up to about 6 degrees below horizon, the contribution of cosmic rays is not exactly zero. This also means that in the case of MAGIC telescopes with FOV of $3^{\circ} \times 3^{\circ}$ the expected signal of tau neutrinos on the camera is not uniform as a function of zenith angle. The camera sees not only the earth crust but also part of the atmosphere or the sea. In principle, pointing to the larger inclinations ( $>95$ degrees) should decrease the fraction of atmosphere seen by the camera and significantly reduce the background level, but in such a case the expected flux of tau neutrinos decreases as well. In [18] it was found that the maximum effective tau neutrino area on the camera is at 91.6 degrees, and reduces almost to zero at 90.5 degrees, and is more than one order of magnitude smaller, in comparison with its maximum value, at $93.5 \mathrm{deg}$. It was also found that the maximum effective neutrino area integrated over the MAGIC FOV is for pointing at 92.5 degrees. This allows to catch the most sensitive part in the outskirts of the camera and add the additional contribution of the effective area above $92.5^{\circ}$. Thus, the seaON data were taken with a zenith angle of $\theta=92.5^{\circ}$.

The software tool used in the present paper to simulate the signatures expected from neutrino-induced showers by MAGIC is based on the code ANIS (All Neutrino Interaction Simulation) [22] with some extension described in [21]. CORSIKA version 6.99 [23], with activated CERENKOV, CURVEDEARTH, TAULEP and THIN options, was used to simulate the shower development of $\tau$-induced showers and its Cherenkov light production. Tau lepton decays have been simulated with PYTHIA version 6.4 [24]. The output of the CORSIKA simulations are then passed to a simulation of the atmospheric extinction and the MAGIC Telescope response [25]. Due to technical difficulties, only showers simulated between 86 degrees and 90 degrees have been used to study the properties of upward-going tau neutrinos. This limitation is not a problem, as the response of IACTs to Cherenkoy light from showers of the same energy and equal column depth depends only slightly on the zenith angle [14]. In our MC, we also performed simulations for inclined showers induced by CRs. This gives the possibility to compare simulated images on the MAGIC camera plane with images from real data.

In the studied energy range, from $1 \mathrm{PeV}$ to $1 \mathrm{EeV}$, the main background for IACTs are proton induced showers. The background from photon and electron induced showers can be expected to be considerably smaller, especially beyond the spectral break in the electron spectrum at about $1 \mathrm{TeV}$ [26].

In the case of showers induced by CRs and observed at large zenith angles, the hadronic and electromagnetic component of extensive air showers (EAS) is almost absorbed [27] because of the deep horizontal column depth of about $\simeq 10^{4}$ up to $5 \times 10^{4}$ $\mathrm{g} \mathrm{cm}^{-2}$ in such directions. In the case of CR showers of lower energy (from tens of $\mathrm{GeV}$ up to $\mathrm{PeV}$ ) only a few pixels in the camera are triggered, yielding dimmer and smaller images (see Figure 2 left). The larger shower images come from high energetic muons (from tens to hundred of $\mathrm{GeV}$ ) produced at the first stages of shower development or muon bundles from later stages. Penetrating muons from bundles can decay into electrons not far from the telescopes, which induce small airshowers producing detectable flashes of Cherenkov light. This produces different image topologies in the camera, e.g. a few clusters of triggered pixels, as is shown in [16]. The estimated event rate for such showers in the case of a MAGIC-like detector is at the level of about one event per two minutes for CR showers of energies above $E_{\mathrm{CR}}>60 \mathrm{PeV}$ [28].

High energy muons $(\mathrm{E}>1 \mathrm{TeV})$ at large zenith angles have an interaction length, via $e^{+} e^{-}$-pair production, bremsstrahlung, and photo-nuclear scattering, comparable to the depth of the atmosphere. In these events produced by interacting muons via radiative processes, additional electromagnetic sub-showers are induced [29, 30]. If these sub-showers are induced close to the detector, it can lead to a strong flash of Cherenkov light and a 
Table 2: Tau lepton decay channels implemented in ANIS simulations. The Table is taken from [6].

\begin{tabular}{cccc}
\hline \hline Decay & Secondaries & Probability & Air-shower \\
\hline \hline$\tau \rightarrow \mu^{-} \bar{v}_{\mu} v_{\tau}$ & $\mu^{-}$ & $17.4 \%$ & weak showers \\
$\tau \rightarrow e^{-} \bar{v}_{e} \nu_{\tau}$ & $e^{-}$ & $17.8 \%$ & 1 Electromagnetic \\
$\tau \rightarrow \pi^{-} v_{\tau}$ & $\pi^{-}$ & $11.8 \%$ & 1 Hadronic \\
$\tau \rightarrow \pi^{-} \pi^{0} v_{\tau}$ & $\pi^{-}, \pi^{0} \rightarrow 2 \gamma$ & $25.8 \%$ & 1 Hadronic, 2 Electromagnetic \\
$\tau \rightarrow \pi^{-} 2 \pi^{0} v_{\tau}$ & $\pi^{-}, 2 \pi^{0} \rightarrow 4 \gamma$ & $10.79 \%$ & 1 Hadronic, 4 Electromagnetic \\
$\tau \rightarrow \pi^{-} 3 \pi^{0} v_{\tau}$ & $\pi^{-}, 3 \pi^{0} \rightarrow 6 \gamma$ & $1.23 \%$ & 1 Hadronic, 6 Electromagnetic \\
$\tau \rightarrow \pi^{-} \pi^{-} \pi^{+} v_{\tau}$ & $2 \pi^{-}, \pi^{+}$ & $10 \%$ & 3 Hadronic \\
$\tau \rightarrow \pi^{-} \pi^{+} \pi^{-} \pi^{0} v_{\tau}$ & $2 \pi^{-}, \pi^{+}, \pi^{0} \rightarrow 2 \gamma$ & $5.18 \%$ & 3 Hadronic, 2 Electromagnetic \\
\hline \hline
\end{tabular}

bright image in the camera (see Figure 2 right). All classes of simulated events shown in Figure 2 and presented also in [16] have been observed in the data taken with the MAGIC telescopes at large zenith.

The expected signature of tau leptons in the camera depends on the different tau decay channels. Tau leptons decay mainly to hadrons, pions and kaons, and in $35 \%$ of the cases to electrons and muons (see Table 2). In [16] we show shower images from our MC signal simulations for a $1 \mathrm{PeV}$ tau lepton decaying into an electron, pion or muon close to the detector, i.e. for a typical detector-to-shower distance of about $50 \mathrm{~km}$. The showers produced by tau leptons have in general larger size and contain many more photons compared to the ones of the background coming from protons. This is not only due to the closer distance to the detector, but also because the tau lepton mostly decays into electrons and pions, which induce electromagnetic subshowers producing a large amount of Cherenkov light. Electromagnetic showers or a superposition of electromagnetic subshowers come from decays of neutral pions, while hadronic sub-showers come from interaction and decay of charged pions. Tau leptons can also decay into muons, see Table 2. At energies of $>1 \mathrm{PeV}$, the muon has a large interaction length of a few thousand kilometers in air, so the muon/mainly interacts with the atmosphere through secondary bremmstrahlung processes [31]. This makes the muon ring image hardly visible in the camera [16] .

\section{Discrimination of $\tau$-induced showers}

Simulated events of $\tau$-induced showers are calibrated following the same procedure as real data. The number of photoelectrons per camera pixel are extracted using a sliding window algorithm [15]. In order to remove the pixels which are most likely due to the night sky background, an image cleaning procedure is carried out [15]. The cleaned camera images are characterized by a set of image parameters which were introduced by Hillas [32].

These parameters provide a geometrical description of the images of showers and are used to infer the energy of the primary particle, its arrival direction, and to distinguish between $\gamma$-ray and hadron induced showers. A typical spatial distribution of Cherenkov photons on the camera can be parameterized as an ellipse. The rms spread of Cherenkov light along the major/minor axis of image is known as the Length/Width of an image. The Length and the Width parameter are a measure of the lateral and the vertical development of the shower. The Size parameter measures the total amount of detected light (in p.e.) in all camera pixels, and is correlated with the primary energy of the shower. In the following we study these parameters also for the case of deep i.e. with the first interaction point deep in the atmosphere, $\tau$-induced simulated showers and compare the corresponding distributions with data.

The MAGIC telescopes took data at zenith angles of 92.5 degrees (seaON) and 87.5 degrees (seaOFF). In the seaOFF data, a negligible signal of neutrinos events is expected. This is due to the fact, that the relative rate of neutrino interactions is lower for seaOFF because of the Earth skimming channel enhances the rate, although this enhancement may not be so high at $1 \mathrm{PeV}$. The seaOFF data is used to estimate the background contribution in the seaON data and also to construct the selection criterion to identify tau-neutrino showers, see [17] for a detailed description.

Here, in Figure 3 (left) we show a 2D distriution of Size versus Length parameter for all seaON data, but in Figure 3 (right) the distribution of the seaON, seaOFF and signal MC events in the direction perpendicular to the selection line. The Y coordinate was obtained from the following formula: $\log _{10}(Y)=$ $\log _{10}($ Size [p.e.] $) * \cos (\alpha)-\log _{10}($ Length $[\mathrm{deg}]) * \sin (\alpha)$, where $\alpha=63.435^{\circ}$. In this representation the selection line shown in Figure 3 (left) transforms to a single cut value given by: $\log _{10}(Y)=2.35$. For showers with impact distance smaller than $0.3 \mathrm{~km}$ we use the following selection cut: $\log _{10}(Y)>2.35$, while for showers with larger impact distance $(0.3-1.3 \mathrm{~km})$ a slightly relaxed cut was used i.e. $\log _{10}(Y)>2.10$. The impact distance is defined as the distance between the shower axis and the telescope axis, see also Figure 6. The distribution of impact distances in CORSIKA simulations can be set by using the option CSCAT [23]. Their reconstruction procedure has been described in [15].

In any case we did not find any neutrino candidate, if this selection criterion is applied to all seaON data, see Figure 3. The selection criterion was optimized by maximization of MC signal efficiency, which finally reached a level of about $40 \%$, and minimization of the background contribution. The selection cut was placed in the $\mathrm{Y}$ range where the signal distribution 


\section{- All seaON data}

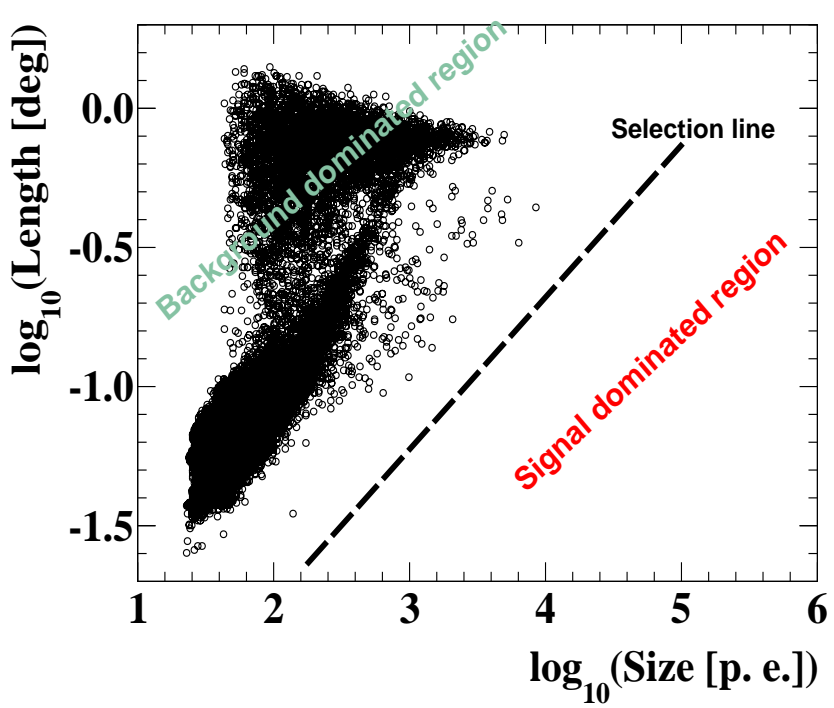

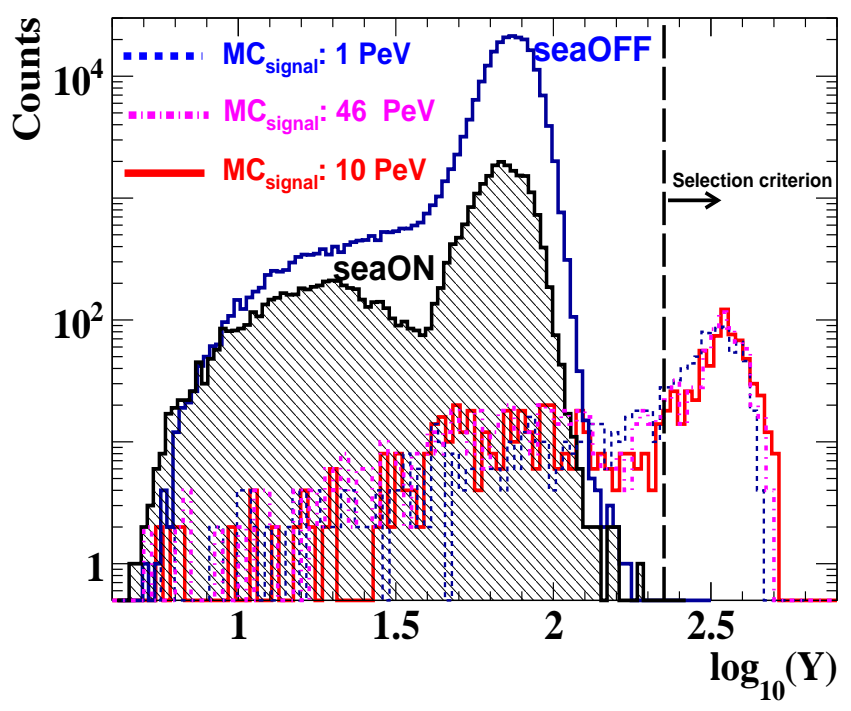

Figure 3: Left panel: 2D distribution of seaON measured events in the Hillas Length versus Size paramater plane. In the signal dominated region, no events remain after selection. Right panel: Distribution of events in the direction perpendicular to the selection line, defined by Y-axis (see text), for seaON, seaOFF and signal MC events. In the region $\log _{10}(Y)>2.35$, no neutrino candidates remain. The selection criterion keeps about $\sim 40 \%$ of MC signal events.
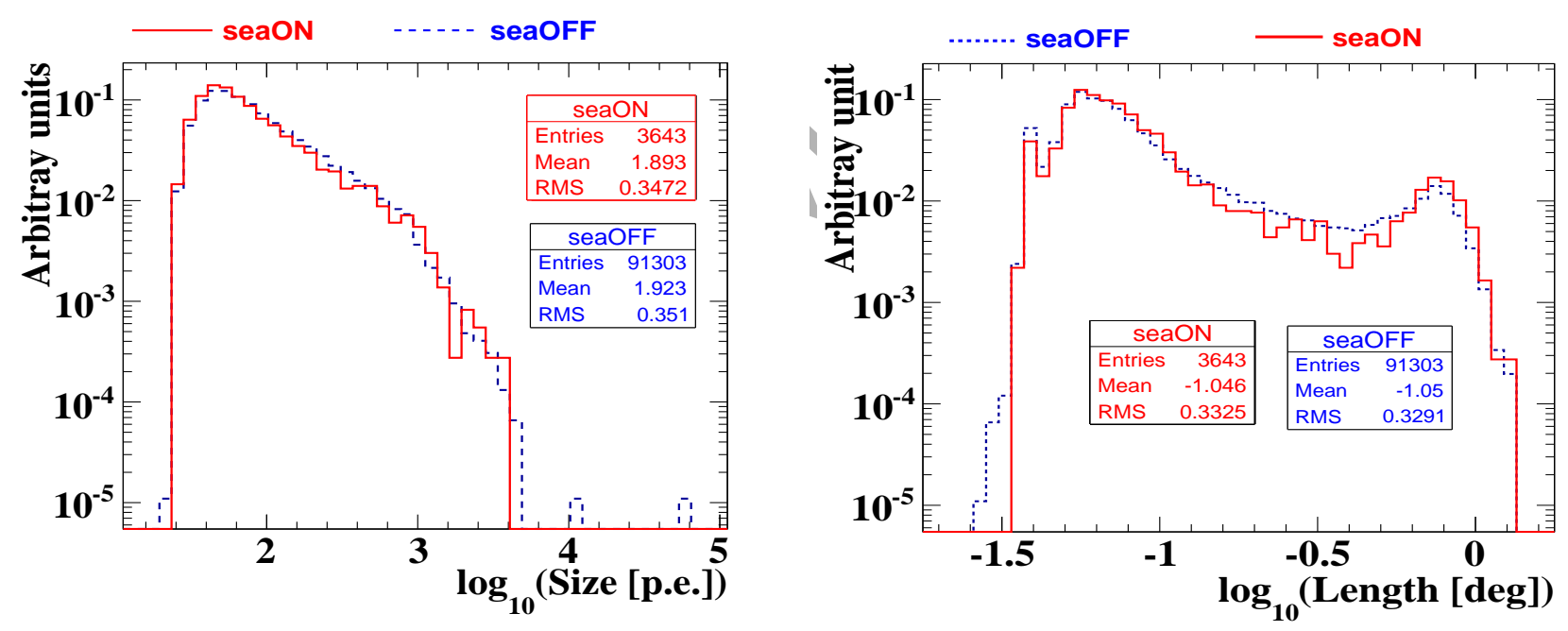

Figure 4: Normalized distribution of the Hillas parameters Size (left panel) and Length (right panel) for seaON and seaOFF data.

starts to be flat, in order to avoid a significant drop of the signal efficiency for larger values of the $\mathrm{Y}$ cut parameter.

As can be seen from the Figure 3 (right) the MC signal distributions look rather similar, as a consequence of the small dependence of the Hillas parameters on the primary energy of the tau lepton, as shown in [16].

It is also worth noting that we see the universal character of the Hillas parameters at large zeniths. This is seen in MC simulations [14] but also in MAGIC data taken during periods of good weather conditions. As an example, in Figure 4 the normalized distribution of Hillas parameters for data taken in the seaOFF $\left(\sim 5.5 \mathrm{hrs}, 87.5^{\circ}\right.$ zenith angle) and seaON ( $6 \mathrm{hrs,}$ $92.5^{\circ}$ zenith angle) direction are shown. The data were taken during one night, under similar weather conditions. As we can see, the Size distribution for seaOFF data is similar compared to seaON data. A similar behavior is seen for the Length parameter. In the case when we merge larger time periods, the shape of the Hillas Size and Length parameters may be slightly different from one night to another breaking this universality behavior. This is due to different weather conditions and thus different attenuation of Cherenkov light. The effect influence a small fraction of low energetic events with $\log _{10}(Y)<1.6$ (see as an example Figure 3, right). Figure 4 and Figure 3 support our previous assumption when performing MC simulations 
only at large zenith angles, in order to study the response of MAGIC for upward-going $\tau$-induced showers.

\section{Event rate estimation}

In this section, we discuss first different models for neutrino production, then show details on the calculations of the acceptance of the MAGIC telescopes, and finally we present the expected sensitivity for $\tau$-induced showers in MAGIC.

\subsection{Astrophysical target flux}

The detection of ultra-high energy neutrinos, with energies in the $\mathrm{PeV}$ range or above, is a topic of great interest in modern astrophysics. The importance comes from the fact that these neutrinos point back to the most energetic particle accelerators in the Universe, and provide information about their underlying acceleration mechanisms. Neutrinos in the $\mathrm{PeV}$ range and above are suspected to be produced by AGNs [33, 34, 35], GRBs [36] or by the interactions of ultra-high-energy cosmic rays with low energy photon backgrounds, such as the cosmic microwave background and the extragalactic background light [37, 38, 39, 40, 41].

The IceCube Collaboration has recently reported the first observation of a cosmic diffuse neutrino flux (all-flavours) in the $100 \mathrm{TeV}$ to $\mathrm{PeV}$ range, which can be described by the power law $[3,42]$ :

$$
\frac{d \Phi_{\nu}\left(E_{v}\right)}{d E_{v}}=\phi \times\left(\frac{E_{v}}{100 \mathrm{TeV}}\right)^{-\gamma}
$$

where $E_{v}$ is energy of neutrino.

The best-fit power law corresponds to a normalization $\phi=$ $6.7_{-1.2}^{+1.1} \times 10^{-18} \mathrm{GeV}^{-1} \mathrm{~cm}^{-2} \mathrm{sr}^{-1} \mathrm{~s}^{-1}$ and $\gamma=2.50 \pm 0.09$. A somewhat harder slope $\gamma=2.2 \pm 0.2$ is found in the muon neutrino signal for upgoing muon neutrinos detected above $\sim 100$ $\mathrm{TeV}$ [43]. The spectrum of the neutrino signal is for the allflavour high-energy starting events with neutrino vertex contained in the detector volume. Individual neutrino sources, however, could not be identified up to now. While many sources of astrophysical origin have been suggested to be responsible for the IceCube signal, like for example star-forming and/or starburst galaxies $[44,45,46,47,48]$ there is not yet enough information to narrow down the possibilities to any particular source or source class. For a review on possible source candidates see $[49,2,3]$.

\subsection{Expected neutrino flux from GRBs}

GRBs are short gamma-ray flashes lasting from fractions of a second to tens of minutes in most cases. During their prompt emission they are the brightest sources in the Universe. GRBs reach an isotropic-equivalent energy of up to $10^{54} \mathrm{ergs}$, and are likely powered by the core-collapse of a very massive star or the merger of two compact objects. The central engine produces highly relativistic collimated jets, which are predicted to host internal shocks, where particles are efficiently accelerated to high energies. In hadronic scenarios accelerated protons interact with ambient synchrotron photons and produce high-energy neutrinos in the PeV-EeV range $[36,50]$. The neutrino emission is expected to be collimated and in temporal coincidence with the prompt gamma-ray emission. However, recent results from the IceCube Collaboration [51] strongly disfavor classical GRBs as sources of the highest energy cosmic rays and neutrinos. Only more complex models assuming multiple emission regions $[52,50]$ can predict a neutrino flux at the level of the IceCube neutrino astrophysical signal. As it is shown in [52], a class of interesting objects are the chocked jet GRBs proposed as a way to model and unify the Low Luminosity GRBs and hypernovae. The basic concept is that a supernova explosion takes place in a dense surrounding medium and the emerging jet is a) completely choked, b) partially choked, with a shock front emerging or c) emerging without obstacles. In case a) we expect only a prompt (duration $10^{1.5} \mathrm{~s}$ ) neutrino emission. In case b) we expect neutrinos from the chocked jet and a delayed $\left(10^{2}-10^{3} \mathrm{~s}\right)$ gamma-ray flare from the shock front, with duration of $>10^{3} \mathrm{~s}$. Case c) predicts prompt and simultaneous neutrino and gamma-ray emission of $10^{3.5} \mathrm{~s}$ duration.

\subsection{Expected neutrino flux from AGNs}

For AGNs the probability of discovering extraterrestrial neutrino point sources varies with the supposed phenomenology of the accelerators and of their emission mechanisms at high energies [33, 34, 35]. Neutrino emission might be possible for sources where charged and neutral mesons are produced simultaneously from hadronic $p-p$ or $p-\gamma$ interactions. These hadronic processes may be present in variable extragalactic objects such as BL Lacs or flat-spectrum radio quasars (FSRQs), as well as in Galactic systems like microquasars and magnetars. Blazars, a subset of radio-loud active galactic nuclei with relativistic jets pointing towards the Earth, can significantly contribute to the diffuse (extragalactic) $\gamma$-ray background [53]. If these $\gamma$-rays originate from proton interactions, the energy budget will be sufficient to account for the intensity of the IceCube neutrino flux [46]. However, a recent stacking analysis using IceCube data suggest that AGN blazars contribute at most $27 \%$ of the observed neutrino intensity [54]. A recent review of a possible correlation between gamma-rays and $\mathrm{PeV}$ neutrinos from blazars can be found in [55].

Nevertheless, flaring AGNs can provide a boosted flux of neutrinos which in some cases could be at the level of the IceCube neutrino signal. In this paper we consider predictions for a sample of generic neutrino fluxes, from photo-hadronic interactions in the case of a few powerful AGNs flares, as shown in Figure 5. Flux-1 and Flux-2 are calculations for the Feb 23, $2006 \gamma$-ray flare of 3C 279 [56]. Flux-3 and Flux-4 represent predictions for PKS 2155-304 in low-state and high-state, respectively [57]. Flux -5 corresponds to a theoretical prediction for 3C 279 calculated in [58].

\subsection{Background estimation}

As already mentioned, high energetic muons or muon bundles can reach the detector and produce large shower images. The muon bundles are the dominant background contribution of the analysis. A possible contribution from showers induced 


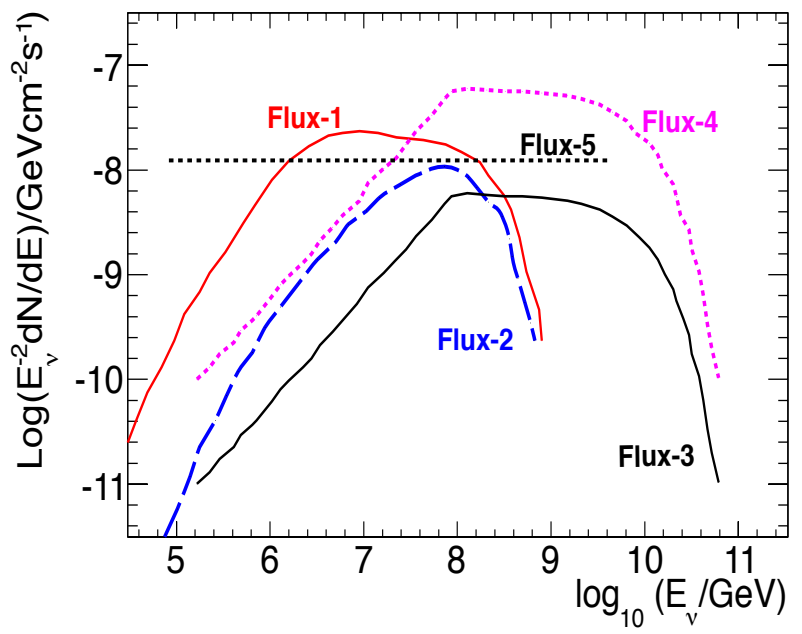

Figure 5: Compilation of the neutrino flux predictions from photo-hadronic interactions in AGNs for the $\gamma$-ray flare of 3C 279 [56] (Flux-1 and Flux 2), PKS 2155-304 [57](Flux-3 and Flux-4) and 3C 279 [58] (Flux-5).

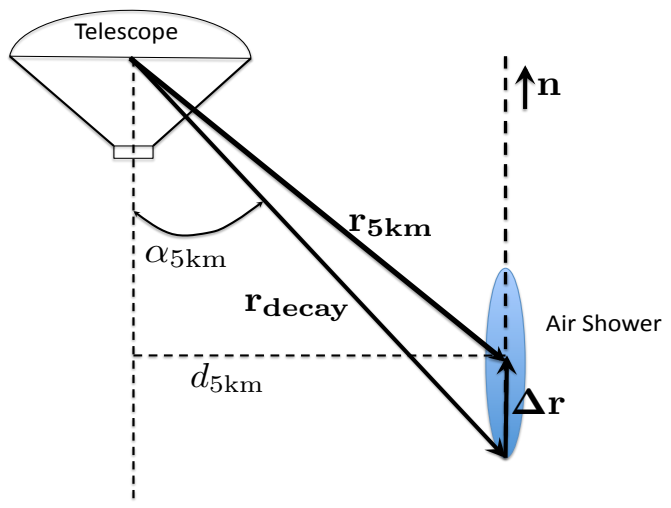

Figure 6: Sketch illustrating the different selections cuts performed in the analysis, to guarantee that the estimated position of the shower maximum of simulated events used in the acceptance calculation is in the FOV of MAGIC (see Section 4.5 for details). The subscript of 5 $\mathrm{km}$ corresponds to estimate position of the shower maximum, which is for shower energies relevant in this analysis i.e. $1 \mathrm{PeV}-3 \mathrm{EeV}$ is reached approximately after $600 \mathrm{~g} / \mathrm{cm}^{2}$ on average, which in the lower part of the atmosphere translates to the distance of about $5 \mathrm{~km}$.

by cosmic ray electrons can be neglected. At horizontal directions the Cherenkov light from electromagnetic showers will be strongly attenuated, even more than for proton primaries of similar energy, and the shower images in the camera will be too small, see e.g. Figure 4 of [14].

In order to calculate the expected number of background events, we used the characterization of the atmospheric muon flux above $15 \mathrm{TeV}$ measured by IceCube [59], which can be modeled by an unbroken power law:

$$
\frac{d \Phi_{\mu}}{d E_{\mu}}=\phi_{\mu} \times\left(\frac{E_{\mu}}{10 \mathrm{TeV}}\right)^{-\delta} .
$$

The values of the parameters that maximize the likelihood for the parametrized muon flux are: $\phi_{\mu}=1.06_{-0.32}^{+0.42} \times$ $10^{-10} \mathrm{TeV}^{-1} \mathrm{~cm}^{-2} \mathrm{sr}^{-1} \mathrm{~s}^{-1}$ and a spectral index of $\delta=3.78 \pm$ 0.02 (stat.) \pm 0.03 (syst.).

This is a conservative assumption, because we do not include here a dependency on the zenith angle which leads to a lower flux of muons at large zenith angles [59]. Using the acceptance with height cut shown in Figure 8 (right panel) and the muon flux given by Eq. 2, the expected background event rate is at a level of $4.3 \times 10^{-7}$ events for one hour of observation.

\subsection{Acceptance of the MAGIC Telescopes}

The propagation of a given neutrino flux through the Earth and the atmosphere is simulated using an extended version of the ANIS code [21]. The extended version gives a possibility to simulate the lepton tau propagation in air for different orographic condition of considered site. For a set of fixed neutrino energies, $10^{6}$ events are generated each on top of the atmosphere for a zenith angle $\theta=92.5^{\circ}$ and an azimuth angle $\phi=-30^{\circ}$. The tau is propagated in small steps until the age of the taulepton exceeds the tau lepton lifetime. The different amount of energy loss in the Earths crust and air have been also taken into account, see [21] for more details. All computations are done using Digital Elevation $\mathrm{Map}^{8}$ to model the surrounding mass distribution of the La Palma site. As a results of these simulations, the flux of leptons emerging from the ground as well as their energy and the decay vertex positions are calculated inside an interaction volume. The interaction volume for a given incoming neutrino with energy $E_{v}$ is defined by a particular plane $A_{\text {gen }}(\theta)$ and distance $\Delta l$,which is a multiple of a few times of the average lepton range. The plane $A_{g e n}(\theta)$ is also the cross-sectional area of the detector volume. The detector volume is modeled by a cylinder with radius of $50 \mathrm{~km}$ and $10 \mathrm{~km}$ height with its $\mathrm{z}$-axis (height) pointing upwards, see Figure 3 in [21]. Since the plane $A_{g e n}(\theta)$ was used as reference plane for the generation of incoming neutrinos, by definition, it is orthogonal to the incoming neutrino direction.

In such an approach the detector aperture/acceptance for an initial neutrino energy $E_{v_{\tau}}$ can be calculated from:

$$
\begin{aligned}
A^{\mathrm{ps}}\left(E_{v_{\tau}}, \theta, \phi\right)= & N\left(E_{v_{\tau}}\right)_{\mathrm{gen}}^{-1} \times \sum_{i=1}^{N_{\text {DISTcut }}} P_{i}\left(E_{v_{\tau}}, E_{\tau}, \theta, \phi\right) \\
& \times A_{\text {gen }, i}(\theta) \times T_{\mathrm{eff}, i}\left(E_{\tau}, r_{5 \mathrm{~km}}, d_{5 \mathrm{~km}}, \theta\right),
\end{aligned}
$$

where $\theta, \phi$ are the simulated zenith and azimuth pointing angles of the MAGIC telescope, and $N_{\text {gen }}\left(E_{\gamma_{\tau}}\right)$ is the number of generated neutrino events per neutrino energy bin. The interaction probability is given by $P\left(E_{v_{\tau}}, E_{\tau}, \theta, \phi\right)$, the probability that

\footnotetext{
${ }^{8}$ Consortium for Spatial Information (CGIAR-CSI) http://srtm.csi. cgiar.org/
} 

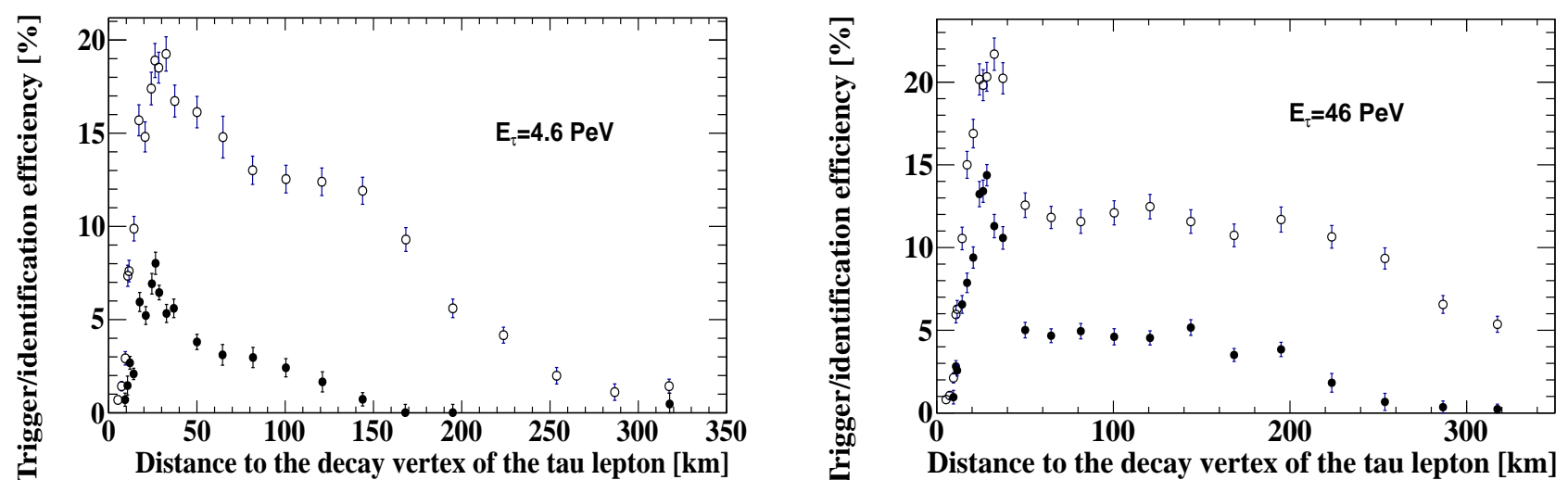

Figure 7: Trigger (open circles) and combined trigger and tau lepton identification efficiency (full circles) for MAGIC as a function of the distance to the decay vertex of the tau lepton, for an energy of $4.6 \mathrm{PeV}$ (left panel) and $46 \mathrm{PeV}$ (right panel). These efficiencies are calculated as an average over simulated showers with an impact distance smaller than $1.3 \mathrm{~km}$ and the DISTcut applied, at a zenith angle $86^{\circ}$. Simulations are presented for equal step size in the vertical depth, i.e. every $20 \mathrm{~g} / \mathrm{cm}^{-2}$.

a neutrino with energy $E_{v_{\tau}}$ and zenith angle $\theta$ and azimuth angle $\phi$ produces a lepton with energy $E_{\tau}$, which can reach the detector volume, see again [21] for more details.

In order to calculate the point source neutrino aperture for the MAGIC, we consider only events which are in the FOV of the MAGIC. Thus, $N_{\text {DISTcut }}$ is the number of $\tau$ leptons with energies $E_{\tau}$ larger than the threshold energy $E_{\text {th }}=1 \mathrm{PeV}$ and after selection cut, which guarantees that the estimated position of the shower maximum lies in the FOV of the MAGIC telescopes. At its maximum, a shower has the largest lateral extension and Cherenkov light production, thus is capable of producing the largest signal seen by IACTs telescopes. The following FOV condition was then used: $\alpha_{5 \mathrm{~km}}=\arcsin \left(d_{5 \mathrm{~km}} / r_{5 \mathrm{~km}}\right)<$ $\left(\delta_{\text {FOV }} / 2+\alpha_{\text {Cher. }}\right) \simeq 3.10^{\circ}$, where $d_{5 \mathrm{~km}}$ is the distance of the estimated shower maximum to the shower axis, the $r_{5} \mathrm{~km}$ is the vector pointing from the telescope to the estimated position of the shower maximum and $\delta_{\mathrm{FOV}}=3.5^{\circ}$ is the FOV of the MAGIC camera, (see Figure 6). In this selection criterion, called here DISTcut, we ensure that a least good fraction of the shower is imaged into the MAGIC camera, see [17] for more detailed desription of this cut

In Eq. $3, T_{\mathrm{eff}, i}\left(E_{\tau}, r_{5 \mathrm{~km}}, d_{5 \mathrm{~km}}, \theta\right)$ is the trigger and reconstruction/cut efficiency for $\tau$-lepton induced showers with its estimated position of the shower maximum at distance $r_{5 \mathrm{~km}}$ from the telescope and the distance $d_{5 \mathrm{~km}}$. In case of the aperture calculations, Eq. 3 was used with $T_{\text {eff, } i}$ set to 1 , while for the acceptance calculations the trigger and identifications cuts are included i.e. $T_{\mathrm{eff}, i}<1$.

The trigger efficiency depends on the response of a given detector and is usually estimated based on MC simulations. The trigger efficiency in an energy interval, $\Delta E$, is defined as the number of the simulated showers with positive trigger decision over the total number of generated showers for fixed zenith angle $\theta$, initial energy of the primary particle $E_{\tau}$, and the impact distance. The impact distance of simulated showers was randomized in the CORSIKA simulations (by using the CSCAT option) and later the Cherenkov telescope orientation for such showers was randomized over the MAGIC camera FOV. In order to eyaluate the identification efficiency for tau neutrino showers we apply in addition the selection criterion shown in Figure 3 (right panel). Figure 7 shows an example of the trigger/identification efficiency for two example energy bins. It is well seen that for smaller distances $\left(r_{\text {decay }}<20 \mathrm{~km}\right)$ the efficiency drops. In such a case the shower maximum is too close to the detector, and the shower does not reach yet the maximum of shower. In general, these plots provide an estimate of the typical distance for $\tau$-induced showers seen by MAGIC.

In Figure 8 (left panel) we show an estimate of the MAGIC point-source aperture (for $T_{\mathrm{eff}, i}=1$ ) to tau neutrinos. The aperture is shown for four cases: (1) for simulations including the orography of the La Palma island, but with the spherical model of Earth, with the rock density of about $2.65 \mathrm{~g} / \mathrm{cm}^{2}$, outside the island, and with $\alpha_{r_{5 \mathrm{~km}}}<3.1^{\circ}$; (2) with an additional impact distance selection criterion $d_{5 \mathrm{~km}}<1.3 \mathrm{~km}$; (3) with a $3.0 \mathrm{~km}$ deep water layer around island ${ }^{9}$; (4) for aperture calculated using a simple analytical approximation (described in Section 4.6). The contribution of the water layer is important, leading to a factor two change in the aperture compared to the simulation which includes the orography of La Palma only, case 1). This is because the $v_{\tau}$ has a much smaller interaction probability in water, and can produce hence smaller escaping $\tau$-lepton fluxes.

An important effect in the analysis is the presence of possible clouds during observations, that needs to be taken into account. At the MAGIC site due to the location of the detector, two classes of clouds can be found: one expected above the MAGIC telescopes and other one below. As mentioned in the introduction the presence of high clouds above the detector can make impossible normal gamma-ray observations but allow to perform horizontal observations for tau neutrinos. The MAGIC lidar system [64] indicates, whenever high clouds in the vertical directions are present at the MAGIC site. This information

\footnotetext{
${ }^{9} \mathrm{~A} 4$ and $4.5 \mathrm{~km}$ deep water layer have been tested as well, which causes a change in the estimated aperture of less than $30 \%$.
} 

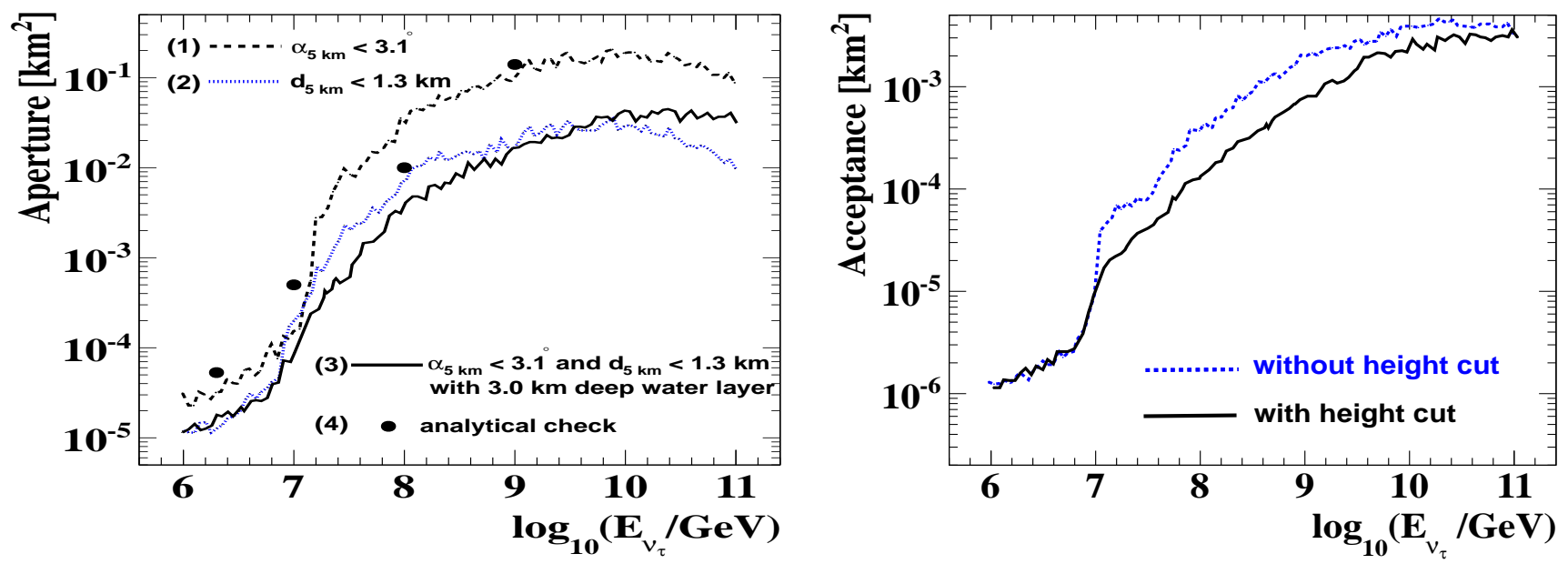

Figure 8: Left: The point-source aperture to earth-skimming tau neutrinos for the MAGIC telescopes pointing at $\theta=92.5^{\circ}$ and $\phi=-30^{\circ}$ for different densities of the interaction medium (see text for more details); Right: The acceptance for point sources, $A^{\mathrm{ps}}\left(E_{v_{\tau}}\right)$ as estimated for the MAGIC site, with a DISTcut of: $\alpha_{5 \mathrm{~km}}<3.1^{\circ}$ and $d_{5 \mathrm{~km}}<1.3 \mathrm{~km}$, within identification efficiencies shown in Figure 7 and with/without the height cut.

is usually used to start observations at large zenith angles, i.e. observations are performed if the transmission for the aerosol component from $9 \mathrm{~km}$ to ground is below 0.55 and from $3 \mathrm{~km}$ to ground is close to 1.0. However, during horizontal observations, we can also expect clouds below the MAGIC telescopes. These clouds usually form the quasi-stable layer of cumulus between $1.5 \mathrm{~km}$ and $1.9 \mathrm{~km}$ a.s.l. due to the temperature inversion at these altitudes [65]. For such case, we did not have any information about clouds present in the directions of the seaON and seaOFF observations, due to the lack of lidar measurements in these directions ${ }^{10}$.

Thus, in our acceptance calculations, as the most conservative case, we assumed the presence of the quasi-stable layer of cumulus between $1.5 \mathrm{~km}$ and $1.9 \mathrm{~km}$ a.s.1., With this assumption all the Cherenkov light generated below $1.9 \mathrm{~km}$ is absorbed. To estimate this effect, all decaying tau leptons below $1.5 \mathrm{~km}$ a.s.l. were discarded (referred to as "height cut"). We assume that in this case, the Cherenkov light is absorbed when it crosses the layer between 1.5 and $1.9 \mathrm{~km}$ a.s.l. This selection provides a conservative upper limit of this effect. Figure 8 (right panel) shows the acceptance obtained with and without the "height cut" applied. As we can see in the plot this selection criterion leads to a smaller (about factor two) acceptance, showing the influence of the quasi-stable layer of cumulus and gives the uncertainty associated to the fact that there are no lidar measurements in the horizontal direction. After simulating the effects of the orography of the site, the sea and the layer of clouds, and also taking into account the trigger and identification efficiency, we obtain an acceptance which is one or two orders (for $10^{18} \mathrm{eV}$ ) of magnitude smaller than calculated aperture.

\footnotetext{
${ }^{10}$ With existing setup, the MAGIC lidar can monitor only clouds layer up to a few tens of kilometers, which is not enough for large zenith angle observations, where we need to know if the clouds are present or not at much larger distances, at least one hundred of kilometers.
}

\subsection{Analytical aperture estimation of the MAGIC Telescopes}

A simple analytical estimate is found to yield the correct order of magnitude for the effective aperture of MAGIC. We focus first on the geometry of the system. As the horizon is observed at a zenith angle of $\theta>90^{\circ}$, the particle path through the Earth is $L \simeq-2 R_{\text {Earth }} \cdot \cos (\theta)$, where $R_{\text {Earth }}$ is the Earth radius, see Figure 1 (left panel). The geometric area seen by telescopes $A_{\text {geom }}$ along the line-of-flight of the neutrino can be approximated by:

$$
\begin{array}{r}
A_{\mathrm{geom}}(\theta)=a \cdot b \cdot \pi=H^{2} \cdot \frac{\delta_{\mathrm{FOV}}}{4} \cdot \pi \cdot\left(\tan \left(\theta+\delta_{\mathrm{FOV}} / 2\right)\right. \\
\left.-\tan \left(\theta-\delta_{\mathrm{FOV}} / 2\right)\right),
\end{array}
$$

where $a$ and $b$ is the major and minor axis of ellipse (see Figure 9), $H=2.2 \mathrm{~km}$ a.s.l. is the altitude of the telescope and $\delta_{\mathrm{FOV}}=0.061 \mathrm{rad}\left(3.5^{\circ}\right)$ is the FOV of the MAGIC camera. The Taylor expansion given in Eq. 4 is accurate within $20 \%$. The ellipse is actually truncated, because the horizon appears already at $\theta_{\text {horizon }}=180^{\circ}-\left(180^{\circ} / \pi \times \arcsin \left(R_{\text {Earth }} /\left(R_{\text {Earth }}+H\right)\right)\right)=91.5$ deg. This effect reduces the geometrical area $A_{\text {geom }}\left(92.5^{\circ}\right)$ from $14.3 \mathrm{~km}^{2}$ to about $5.33 \mathrm{~km}^{2}$.

The conversion efficiency for tau neutrinos along the distance $L$ is calculated from [66]:

$$
\begin{aligned}
P\left(E_{v_{\tau}}, E_{\tau}, \theta\right)= & \int_{0}^{L} \exp \left(-x / \lambda_{v_{\tau}}\right) \cdot \exp \left(-(L-x) / \lambda_{\tau}\right) \cdot \frac{d x}{\lambda_{v_{\tau}}} \\
& =\frac{\lambda_{\tau}}{\lambda_{v_{\tau}}-\lambda_{\tau}} \cdot\left(\exp \left(-L / \lambda_{v_{\tau}}\right)-\exp \left(-L / \lambda_{\tau}\right)\right),
\end{aligned}
$$

where $\lambda_{v}=1 /\left(\sigma_{\mathrm{CC}} N_{A} \rho\right)$ is the neutrino mean free path, $N_{A}$ the Avogadro constant, $\sigma_{\mathrm{CC}}$ the charged current neutrino interaction cross-section [72] and $\rho$ the density of the rock. The decay length of the tau particle $\lambda_{\tau}=48910 \mathrm{~m} \times\left(E_{\tau} / 1000 \mathrm{PeV}\right)$. The effective area can be estimated as $A\left(E_{v_{\tau}}, \theta\right)=P\left(E_{v_{\tau}}, E_{\tau}, \theta\right) \times$ $A_{\text {geom }}(\theta)$, under the approximation that all tau leptons decay close to the sea surface, which is true only for tau lepton energies below $\sim 30 \mathrm{PeV}$, and without taking into account trigger 
Table 3: Comparison of the aperture $\left(A^{p s}\left(E_{v_{\tau}}\right)\right)$ from MC simulations (Section 4.5) and an analytical approach (Section 4.6). Results are obtained for a zenith angle of $92.5^{\circ}$, neutrino crossing distance $L=546 \mathrm{~km}$, an average rock density $\rho=2.65 \mathrm{~g} / \mathrm{cm}^{2}$ and the charged current neutrino cross-section from [72]. The energy of the tau particle is approximated as $E_{\tau}=0.75 E_{\nu_{\tau}}$.

\begin{tabular}{lccccccc}
\hline \hline $\begin{array}{l}E_{v_{\tau}} \\
(\mathrm{PeV})\end{array}$ & $\begin{array}{c}\sigma_{C C}\left(E_{v_{\tau}}\right) \\
(\mathrm{pb})\end{array}$ & $\begin{array}{c}\lambda_{v_{\tau}} \\
(\mathrm{km})\end{array}$ & $\begin{array}{c}\lambda_{\tau} \\
(\mathrm{km})\end{array}$ & $P\left(E_{v_{\tau}}, E_{\tau}, \theta\right)$ & $\begin{array}{c}A_{\text {geom }} \\
\left(\mathrm{km}^{2}\right)\end{array}$ & $\begin{array}{c}A\left(E_{v_{\tau}}\right) \\
\left(\mathrm{km}^{2}\right)\end{array}$ & $\begin{array}{c}A^{p s}\left(E_{v_{\tau}}\right) \\
\left(\mathrm{km}^{2}\right)\end{array}$ \\
\hline \hline 2 & 950 & 6596 & 0.073 & $1.0 \times 10^{-5}$ & 5.33 & $5.3 \times 10^{-5}$ & $1.5 \times 10^{-4}$ \\
10 & 1900 & 3298 & 0.367 & $9.4 \times 10^{-5}$ & 5.33 & $5.0 \times 10^{-4}$ & $2.7 \times 10^{-4}$ \\
100 & 4800 & 1305 & 3.670 & $1.9 \times 10^{-3}$ & 5.33 & $1.0 \times 10^{-2}$ & $8.0 \times 10^{-3}$ \\
1000 & 11000 & 569 & 36.70 & $2.6 \times 10^{-2}$ & 5.33 & $1.4 \times 10^{-1}$ & $2.6 \times 10^{-2}$ \\
\hline \hline
\end{tabular}

and cut efficiencies. If a tau lepton above an energy of a few tens of PeV still escapes the sea, its decay length can be too large to initiate an air shower before reaching the telescopes. In Table 3 ,

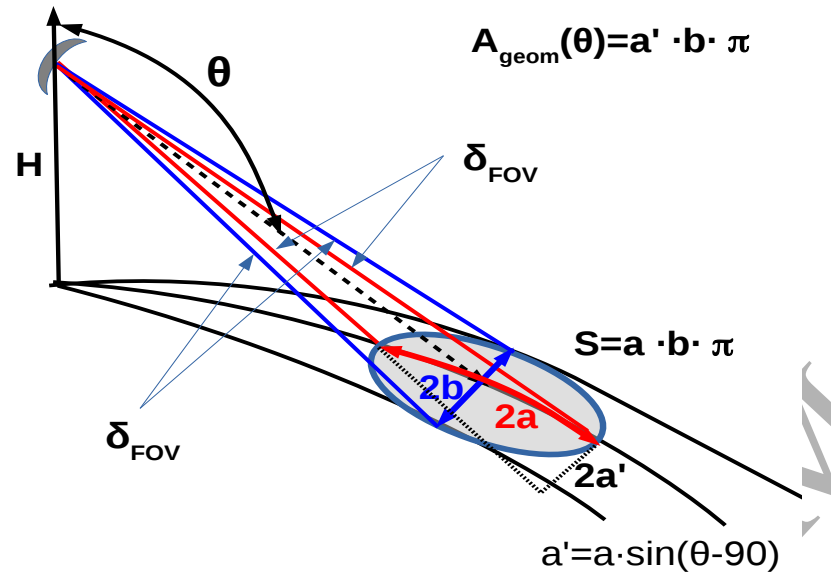

$\mathrm{b}=\mathrm{H} \cdot \delta_{\mathrm{FOV}} /(2 \cdot \cos (180-\theta))$

$\mathrm{a}=\mathrm{H} / 2 \cdot\left(\tan \left(180-\theta+\delta_{\mathrm{FOV}} / 2\right)-\tan \left(180-\theta-\delta_{\mathrm{FOV}} / 2\right)\right)$

Figure 9: The geometrical area opened by the FOV of the MAGIC telescopes.

the conversion efficiencies and comparison of the effective area $\left(A\left(E_{v_{\tau}}\right)\right)$ with our MC estimate $\left(A^{p s}\left(E_{v_{\tau}}\right)\right)$ are shown for five energies. The simple analytical calculations agree (apart from the last point at $1000 \mathrm{PeV}$ ), with the aperture estimate from $\mathrm{MC}$ simulations within a factor of 2 (see Figure 8, left).

\subsection{Event rates}

The total number of expected signal events $\mathrm{N}$ is obtained as:

$$
N=\Delta T \times \int_{E_{\mathrm{th}}}^{E_{\max }} A^{\mathrm{ps}}\left(E_{v_{\tau}}\right) \times \Phi\left(E_{v_{\tau}}\right) \times d E_{v_{\tau}},
$$

where $\Delta T$ is the observation time, $A^{\mathrm{ps}}\left(E_{v_{\tau}}\right)$ the point source acceptance and $\Phi\left(E_{v_{\tau}}\right)$ the expected neutrino flux. Since $A^{\mathrm{ps}}\left(E_{\gamma_{\tau}}\right)$ depends on the zenith angle and hence on time (since all sources move in the sky), in reality, an integral from 0 to $\Delta T$ must be made over time $t$, and $A^{\mathrm{ps}}\left(E_{v_{\tau}}\right)$ should be replaced by $A^{\mathrm{ps}}\left(E_{v_{\tau}}, t\right)$. Thus, all numbers presented in the following have to be considered approximations since they used a time-independent value of $A^{\mathrm{ps}}\left(E_{v_{\tau}}\right)$.

Here we provide an estimate of the event rate for a sample of generic neutrino fluxes, from photo-hadronic interactions in case of flaring AGNs, if observed at the most efficient zenith angle. Flaring AGNs can produce a boosted flux of neutrinos. Table 4 shows the expected event rates for MAGIC, using the flux benchmark models shown in Figure 5.

The rates are calculated for tau neutrinos assuming that the source is in the MAGIC FOV for a period of 3 hours and with the acceptance calculated with and without the "height cut" applied. In the case of Flux-3 and Flux-4 for events with energies of the $\sim 10^{8} \mathrm{GeV}$, the expected event rate with the "height cut" applied is of the order $3 \times 10^{-5}$ events per 3 hours. In the case of Flux-1, Flux-2 and Flux-5, for events below $\sim 5 \times 10^{7} \mathrm{GeV}$, the number of expected events is below $1.1 \times 10^{-5}$ in 3 hours. In case the "height cut" selection is not applied, the expected number of events increases by a factor 2 . We give results for both cases, because sometimes it is also possible that observations can be performed during absence of quasi-stable layer of cumulus in seaON direction.

The systematic uncertainties on the event rates due to the tau-lepton energy loss and the neutrino-nucleon cross section have been taken into account. The average energy loss of tau particles per distance travelled (unit depth $X$ in $\mathrm{g} \mathrm{cm}^{-2}$ ) can be described as $\langle d E / d X\rangle=\alpha(E)+\beta(E) E$. The factor $\alpha(E)$, which is nearly constant, is due to ionization. $\beta(E)$ is the sum of $e^{+} e^{-}$-pair production and bremsstrahlung and photonuclear scattering, which is not only the dominant contribution at high energies but at the same time subject to relatively large uncertainties. In this work, the factor $\beta_{\tau}$ is calculated using the following models describing the contribution of photonuclear scattering: ALLM [62], BB/BS [68], CMKT [69], and different neutrino-nucleon cross-sections: GRV981o [76], CTEQ66c [70], HP [71], ASSS [72], ASW [73]. The results are listed in Table 5 for Flux- 1 and Flux-3, and show that the combined systematic uncertainty of both effects can be considerable, namely of the order of $40 \%$, but nevertheless much smaller than the effect of the quasi-stable layer of cumulus below the observatory, included in the "height cut", which can lead to a factor two of the lower event rate. 
Table 4: Expected event rates in the MAGIC telescopes for AGN flares with the Flux predictions described in Section 4.3.

\begin{tabular}{lcccccc}
\hline \hline & $\begin{array}{c}\text { Flux-1 } \\
\left(\times 10^{-5} / 3 \text { hrs }\right)\end{array}$ & $\begin{array}{c}\text { Flux-2 } \\
\left(\times 10^{-5} / 3 \text { hrs }\right)\end{array}$ & $\begin{array}{c}\text { Flux-3 } \\
\left(\times 10^{-5} / 3 \text { hrs }\right)\end{array}$ & $\begin{array}{c}\text { Flux-4 } \\
\left(\times 10^{-5} / 3 \text { hrs }\right)\end{array}$ & $\begin{array}{c}\text { Flux-5 } \\
\left(\times 10^{-5} / 3 \text { hrs }\right)\end{array}$ \\
\hline \hline$N_{\text {Events }}$ & without height cut & 2.4 & 1.4 & 0.74 & 7.4 & 2.4 \\
$N_{\text {Events }}$ & with height cut & 1.1 & 0.6 & 0.30 & 2.9 & 1.2 \\
\hline \hline
\end{tabular}
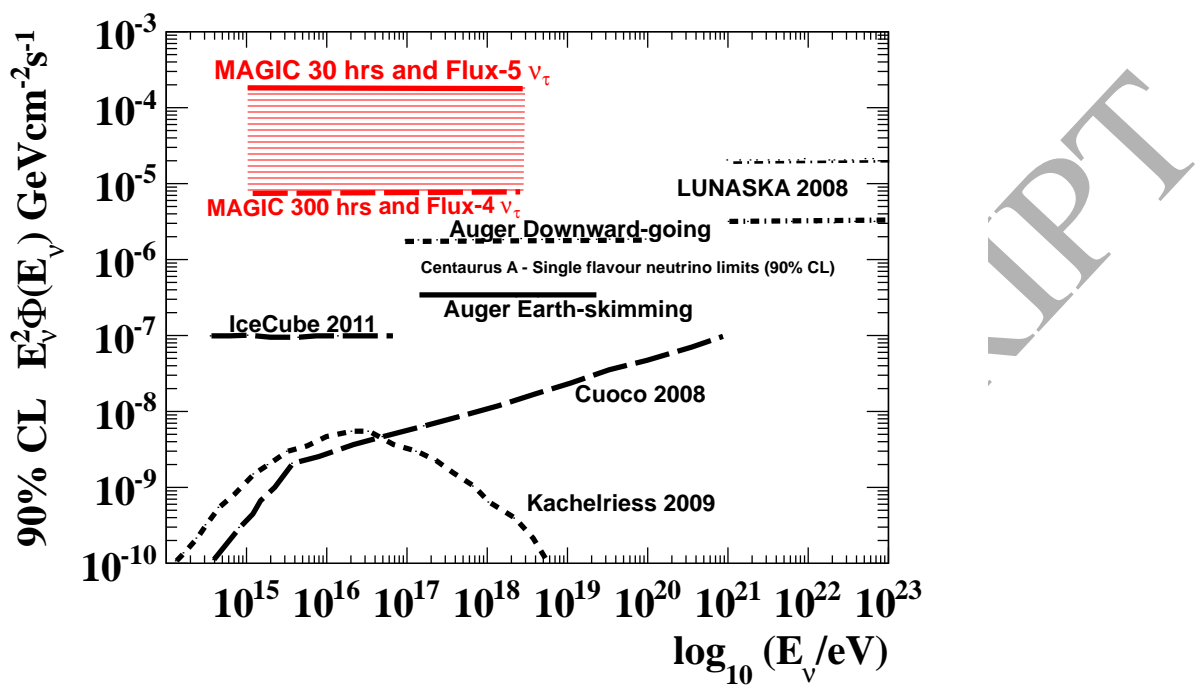

Figure 10: $90 \%$ C.L. upper limit on the tau neutrino flux obtained with the MAGIC telescopes with 30 hrs of observation (red solid line) assuming Flux-5 (see Figure 5). The expected upper limit with 300 hrs of observations assuming Flux-4, is shown as the red dashed line. The results are compared to the $90 \%$ C.L. upper limit on the single flavour neutrino flux from Pierre Auger [63], IceCube [61] and LUNASKA 2008 [77]. The predicted fluxes for two theoretical models of ultra high neutrinos production in the jets [75] and close to the core of Centaurus A [78] are also shown for comparison. Plots adopted from [63].

Table 5: Relative contributions to the systematic uncertainties on the up-going tau neutrino rate. Systematic uncertainty on the expected tau neutrino rate due the neutrino-nucleon cross section and the tau-lepton energy loss. Both uncertainties have been added in quadrature. As a reference GRV981o and ALLM model for Flux-1 and Flux-3 was used.

\begin{tabular}{|c|c|c|}
\hline model & cross-section $\quad \beta_{\tau}$ & Total \\
\hline Flux-1 & $\begin{array}{l}+14 \% \\
-2 \%\end{array}$ & $\begin{array}{l}+14 \% \\
-7 \%\end{array}$ \\
\hline Flux-3 & $\begin{array}{ll}+42 \% & +7 \% \\
-7 \% & \end{array}$ & $\begin{array}{l}+43 \% \\
-16 \%\end{array}$ \\
\hline
\end{tabular}

\section{Tau neutrino flux limit}

From the estimated acceptance with height cut, the sensitivity for an injected spectrum $K \times \Phi\left(E_{v}\right)$ with a known shape $\Phi\left(E_{v}\right)$ was calculated. As no events survived after event selection, 90\% C.L. upper limits [74] on the tau neutrino flux have been obtained. Assuming a reference spectrum of $\Phi\left(E_{v}\right)=$ $1 \times 10^{-8} E^{-2} \mathrm{GeV}^{-1} \mathrm{~cm}^{-2} \mathrm{~s}^{-1}$ of a point-like source, the upper limit obtained is: $K_{90 \%}=2.44 / N_{\text {Events. }}$. The limit for a point source search is then:

$$
E_{v_{\tau}}^{2} \Phi^{p s}\left(E_{v_{\tau}}\right)<2.0 \times 10^{-4} \mathrm{GeV} \mathrm{cm}^{-2} \mathrm{~s}^{-1}
$$

where $E_{v_{\tau}}$ is in the range between 1 and $3000 \mathrm{PeV}$. The neutrino flux upper limit is obtained for an expected number of tau neutrino events of $N_{\text {Events }}=1.2 \times 10^{-4}$, in the case of Flux-5, and is shown in Figure 10 (solid red line). The result is also compared to the $90 \%$ C.L. upper limit on the single flavor neutrino flux from the Pierre Auger experiment [63] from the active galaxy Centaurus A. The expected MAGIC limit could be improved in the case of 300 hours of observations during a strong flare as in Flux-4, where a limit of $E_{v_{\tau}}^{2} \Phi^{p s}\left(E_{v_{\tau}}\right)<8.4 \times 10^{-6}$ $\mathrm{GeV} \mathrm{cm} \mathrm{c}^{-2} \mathrm{~s}^{-1}$ can be obtained. This expectation is shown in Figure 10 as the dashed red line, and is only a factor 3.4 worse than the Pierre-Auger "down-going" analysis.

\section{Summary}

In this paper, a search for tau neutrinos of astrophysical origin in the energy range between $1 \mathrm{PeV}$ and $3 \mathrm{EeV}$ with the MAGIC telescopes is presented. The data was collected during a special pointing of the telescopes below the horizon, to detect Earth-skimming tau-lepton induced showers. These observations can take place during periods of high clouds, which prevent standard gamma ray observations. A $90 \%$ C.L. upper limit on the tau-neutrino flux of $E_{v_{\tau}}^{2} \Phi^{p s}\left(E_{v_{\tau}}\right)<2.0 \times 10^{-4} \mathrm{GeV} \mathrm{cm}^{-2}$ $\mathrm{s}^{-1}$ was obtained, with 30 hours of observation. The limit is 
not competitive with other experiments, hovewer to our knowledge this is first time that is has been calculated with realistics assumptions and using backround data collected by MAGIC. Thus our search gives a realistic illustration of the potential of the Cherenkov technique for this present active topic of research. The presented results can also be important for future Cherenkov experiments like for example the Cherenkov Telescope Array. This next generation ground-base observatory can have a much better possibility to detect tau neutrinos, given its a larger FOV (e.g. in extended observation mode) and much larger effective area

\section{Acknowledgments}

We would like to thank the Instituto de Astrofísica de Canarias for the excellent working conditions at the Observatorio del Roque de los Muchachos in La Palma. The financial support of the German BMBF and MPG, the Italian INFN and INAF, the Swiss National Fund SNF, the ERDF under the Spanish MINECO (FPA2015-69818-P, FPA201236668, FPA2015-68378-P, FPA2015-69210-C6-2-R, FPA201569210-C6-4-R, FPA2015-69210-C6-6-R, AYA2015-71042-P, AYA2016-76012-C3-1-P, ESP2015-71662-C2-2-P, CSD200900064), and the Japanese JSPS and MEXT is gratefully acknowledged. This work was also supported by the Spanish Centro de Excelencia "Severo Ochoa" SEV-2012-0234 and SEV-2015-0548, and Unidad de Excelencia "María de Maeztu" MDM-2014-0369, by the Croatian Science Foundation (HrZZ) Project IP-2016-06-9782 and the University of Rijeka Project 13.12.1.3.02, by the DFG Collaborative Research Centers SFB823/C4 and SFB876/C3, the Polish National Research Centre grant UMO-2016/22/M/ST9/00382 and by the Brazilian MCTIC, CNPq and FAPERJ.

\section{References}

\section{References}

[1] M.G. Aartsen, et al., Phys. Rev. Lett., 113 (2014) 101101 [arxiv:1405.5303].

[2] M.G. Aartsen, et al., Phys. Rev. Lett., 114 (2015) 171102.

[3] M.G. Aartsen, et al., ApJ, 8091 (2015) 98.

[4] A. Aab, et al., Phys. Rev. D, 91 (2015) 092008 [astro-ph 1504.05397].

[5] J.H. Adams, et al., Experimental Astronomy, 40 (2015) 315 .

[6] D. Fargion, ApJ, 570 (2002) 909 [astro-ph 0002453].

[7] J.J. Feng, P. Fisher, F. Wilczek, and T.M. Yu, Phys. Rev. Lett., 88 (2002) 161102 [hep-ph/0105067].

[8] X. Bertou, P. Billoir, O. Deligny, C. Lachaud and A. Letessier-Selvon, Astropart. Phys., 17 (2002) 183 [astro-ph/0104452].

[9] J. Jones, I. Mocioiu, M.H. Reno, and I. Sarcevic, Phys. Rev. D, 69 (2004) 033004.

[10] E. Reya, and J. Rodiger, Phys. Rev. D, 72 (2005) 053004.

[11] Y. Asaoka, and M. Sasaki, Astropart. Phys. 41 (2013) 7.

[12] M.S. Sasaki, et al., (2014) [arXiv:1408.6244].

[13] D. Góra, et al. 2015, Astropart. Phys., 61 (2015) 12.

[14] D. Góra, and E. Bernardini,. Astropart. Phys., 82 (2016) 77.

[15] J. Aleksić, et al., Astropart. Phys., 72 (2016) 76.

[16] D. Góra et. al., Proc of 35th I.C.R.C. (Busan) (2017), POS(ICRC2017)992 [arXiv:1708.06147].

[17] D. Góra et. al., Proc of EPS-HEP (Venice) (2017),PoS(EPSHEP2017)017 [arXiv:1710.04165].
[18] M. Gaug, C. Hsu, J.K. Becker, et al., Proc of 30th I.C.R.C. (Merida) (2007) 1273.

[19] S. Cavazzani, et al., MNRAS 4112 (2011) 1271.

[20] ATel \#: 7856; http://www.astronomerstelegram.org/?read=7856

[21] D. Góra, M. Roth, and A. Tamburro, Astropart. Phys., 26 (2007) 402.

[22] A.Z. Gazizow, and M. Kowalski, Comput. Phys. Commun.,172 (2005) 203.

[23] D. Heck, J. Knapp, J.N. Capdevielle, G. Schatz, T. Thouw, Report FZKA (1998) 6019.

[24] T. Sjöstrand, S. Mrenna, and P. Skands, JHEP 0605 (2006) 026.

[25] R. Zanin, et al., Proc of 33rd I.C.R.C. (Rio de Janeiro) (2013).

[26] F. Aharonian, et al., Phys. Rev. Lett., 101 (2008) 261104.

[27] I. Valino, et al., Astropart. Phys., 32 (2010) 304.

[28] D. Fargion, Prog. Part. Nucl. Phys., 57 (2006) 384

[29] P. Kiraly, et al., J. Phys., A4 (1971) 367.

[30] A.N. Cillis, and S.J. Sciutto, Phys. Rev. D, 64 (2001) 013010.

[31] M. Ambrosio, et al. (2003) [arXiv:astro-ph/0302602].

[32] M.A. Hillas, Nucl. Phys. Proc. Suppl., 52B (1997) 29.

[33] A.M. Atoyan,C.D. Dermer, New Astron. Rey., 48 (2004) 381.

[34] A. Neronov, and M. Ribordy, Phys. Rev. D, 80 (2009) 083008.

[35] A. Mucke, et al., Astropart. Phys., 186 (2003) 593.

[36] E. Waxman, and J. Bahealt, Phys. Rev. Lett., 78 (1997) 2292; K. Murase, and S. Nagataki, Phys. Rev. D, 73 (2006) 063002.

[37] V.S. Berezinsky, and G. Zatsepin, Phys. Lett. B, 28 (1969) 423.

[38] F.W. Stecker, Astrophys. Space Sci., 20 (1973) 47.

[39] V.S. Berezinsky, and A.I. Smirnov, Astrophys. Space Sci., 32, (1975) 461.

[40] R. Engel, D. Seckel, and T. Stanev, Phys. Rev. D, 64 (2001) 093010 [astro$\mathrm{ph} / 0101216]$.

[41] D.V. Semikoz, and G. Sigl, JCAP 4 (2004) 003 [hep-ph/0309328].

[42] C. Kopper, et al., PoS(ICRC2017) (2017) 981 [arXiv:1710.01191 p. 54]

[43] M.G. Aartsen, et al., Phys. Rev. Lett., 115 (2015) 081102 [arxiv: 1507.04005].

[44] G. Giacinti, M. Kachelriess, O. Kalashev, D.V. Semikoz, and A.N Neronov, Phys. Rev. D, 92 (2015) 083016 [astro-ph:1507.07534]

[45] A. Loeb, and E. Waxman, JCAP, 5 (2006) 003 [astroph/0601695]

[46] K. Murase, M. Ahlers, M., and B.C. Lacki, Phys. Rev. D, 88 (2013) 121301, [arXiv:1306.3417]

[47] I. Tamborra, S. Ando, and K. Murase, JCAP 9 (2014) 043, [astro-ph: 1404.1189].

[48] K. Bechtol, M. Ahlers, M. Di Mauro, M. Ajello, and J. Vandenbroucke, ApJ, 83647 (2017) 9 [astro-ph:1511.00688].

[49] L.A. Anchordoqui, et al., Journal of High Energy Astrophysics, 1-2 (2014) 1.

[50] M. Bustamante, K. Murase, and W. Winter, ApJ, 833 (2017) 17 [arXiv: 1606.02325].

[51] M.G. Aartsen, et al., ApJ, 8242 (2016) 115.

[52] N. Senno, K. Murase, P. Meszaros, Phys. Rev. D, 93 (2016) 083003 [arxiv:1512.08513].

[53] M. Ajello, D. Gasparrini, M. , Snchez-Conde, et al., Astrophysical Journal Letters, 800 (2015) L27.

[54] T. Glusenkamp, EPJ Web Conf., 121 (2016) 05006.

[55] S. Gao,M. Pohl, and W. Winter, ApJ, 843, 2 (2017) 19 [astro-ph 1610.05306].

[56] A. Reimer, Int. Journ. of Mod. Phys. D, 18 (2009) 1511.

[57] K. Becker, K. et al., Nucl. Instr. and Meth. in Phys. Res. Sect. A, 630, 1 (2011) 269

[58] A.M. Atoyan, and C.D. Dermer, Phys. Rev. Lett., 87 (2001) 221102.

[59] M.G. Aartsen, et al, Astropart. Phys., 78 (2016) 1.

[60] K.H. Kampert., and M. Unger, Astropart. Phys. 35, 10 (2012) 660 [arXiv:1201.0018].

[61] R. Abbasi, Y. Abdou, T. Abu-Zayyad, et al., ApJ, 732 (2011) 18.

[62] H. Abramowicz, and A. Levy, 1997 [hep-ph/9712415]

[63] P. Abreu, et al. 2012, The Astrophysical Journal Letters, 755 (2012) L4 [arXiv: 1210.3143].

[64] Ch. Fruck, et al.. Proc of 33rd I.C.R.C. (Rio de Janeiro) (2013) [arXiv:1403.3591].

[65] J. Carrillo, et al., Boundary-Layer Meteorology, 158 (2016) 311.

[66] G.W.S. Hou, and M.A. Huang, Proc, of NCTS Work. on Astropart. Phys., Taiwan (2001) [astro-ph:0204145].

[72] A. Cooper-Sarkar,P. Mertsch, S. Sarkar, JHEP, 08 (2011) 042.

[68] E.V. Bugaev, and Y.V. Shlepin, Phys. Rev. D, 67, (3) (2003) 034027. 
[69] A. Cappella, A. Kaidalov, C. Merino, J. Tran Thanh, Phys. Lett. B, 337, (1994) 358

[70] H. Lai, et al. 2000, Eur. Phys. J., C12 (2000) 375 [hep-ph/9903282].

[71] A.Z. Gazizov, and S.I. Yanush, Phys. Rev. D, 65 (9) (2002) 093003.

[72] A. Cooper-Sarkar,P. Mertsch, S. Sarkar, JHEP, 08 (2011) 042.

[73] J.L. Albacete, N. Armesto, J.G. Milhano, C.A. Salgado, U.A. Wiedemann, Eur. Phys. J., C43 (2005) 353.

[74] G.J. Feldman, and R.D. Cousins, Phys. Rev. D, 57 (1998) 3889.

[75] A. Cuoco, S. Hannestad, Phys. Rev. D, 78 (2008) 023007.

[76] M. Gluck, E. Reya, and A. Vogt, Eur. Phys. J., C5 (1998) 461.

[77] C.W. James,R.J. Protheroe, R.D. Ekers, et al., MNRAS, 410 (2011) 885.

[78] M. Kachelriess, S. Ostapchenko., and R. Tomas, New J. Phys., 11, (2009) 065017.

[79] A. Kusenko, and T.J. Weiler, Phys. Rev. Lett., 86, 16 (2002) 161101.

[80] H.L. Vargas, A.S.E. Belmont, and R. Alfaro, Advances in Astronomy, (2017) 1932413 [astro-ph 1610.04820]. 